\title{
Measuring Concentration of Distances -An Effective and Efficient Empirical Index
}

\author{
Sushma Kumari, Balasubramaniam Jayaram, Member, IEEE
}

\begin{abstract}
High dimensional data analysis gives rise to many challenges. One such that has come to gain a lot of attention recently is the concentration of distances (CoD) phenomenon, which is the inability of distance functions to distinguish points well in high dimensions. CoD affects almost every machine learning and data analysis algorithm in high dimensions. In this work, we present a novel efficient and effective empirical index that not only illustrates whether a distance function tends to concentrate for a given data set, but also enables us to measure the rate of concentration and allows us to compare different distance functions vis-á-vis their rate of concentration. As opposed to existing empirical indices, the proposed empirical measure uses only the internal characteristics of a given data set and hence is applicable on real data sets, which was hitherto not possible.
\end{abstract}

Index Terms-Dimensionality Curse, Concentration of Distances, Concentration Function, Dispersion Function.

\section{Introduction}

The term 'Big Data' has come to be related to any data whose characterics can be classified among one of the following 5 V's: Volume, Variety, Velocity, Veracity and Value. While the first of the five V's, namely, Volume is largely taken to refer to the amount or size of data, yet another aspect related to volume is that of Dimensionality [39].

Data sets grow in their complexity not only due to their size but also due to the addition of more features or dimensions to the data. Given a data set $\mathcal{X}$, while Volume refers to the cardinality $\sharp \mathcal{X}$ of the data set $\mathcal{X}$, dimensionality refers to the space in which $\mathcal{X}$ itself is embedded.

Evolution of new data types such as images, videos, audio, gene expression data, etc., lead us to work with data in high dimension, thus forcing us to deal with the so called Dimensionality Curse (DC), a term that has come to refer to some non-intuitive phenomena that occur while dealing with data in high dimensions.

\subsection{The Dimensionality Curse}

The term curse of dimensionality, was first introduced by Bellman [5] while discussing optimisation problems involving high dimensions. However, recently, this term has come to denote or refer to many, often counterintuitive, challenges faced in high dimensions.

There are many aspects of the DC and their effects are still only being explored and is currently a hot topic

S. Kumari is currently with the Department of Mathematics, Kyoto University, Japan and B. Jayaram is at the Department of Mathematics, Indian Institute of Technology Hyderabad, Andhra Pradesh - 502 205, India. This work was done when S. Kumari was at IIT Hyderabad, India. e-mail: ma13m1011@iith.ac.in (S. Kumari), jbala@iith.ac.in (B. Jayaram). of research. This is also clear from the many papers that continue to appear, see for instance, [2], [8], [23], [31]. Two of the well-known aspects of the DC are:

(i) Combinatorial explosion in Search Space, where the search space grows exponentially due to the increase in the number of variables [5].

(ii) Hughes Phenomenon - which refers to the need for at least a sub-exponential growth in the number of data points as dimension increases for many of the data analysis algorithms to be consistent, see for instance, [19], [27].

However, recently, many other aspects of the DC have also been discovered and are being investigated. For instance, the Hubness Phenomenon, which was firstly reported by [4] and later on investigated by Radovanovic et al. [28], [29], [36], which refers to the formation of hubs, i.e., a subset of data points which are more popular as nearest neighbors than other data points.

Yet another major aspect of the DC that has recently come to the fore is the Concentration of Distances phenomenon, which will form the main focus of this work.

\subsection{Concentration of Distances}

Concentration of Distances (CoD), also referred to as Concentration of Norms in the literature, refers to the inability of distance functions to distinguish points well in high dimensions. To measure the closeness between any two objects/points we need the concept of a distance or its dual concept of similarity. However, as the dimension increases all the points appear to be approximately at the same distance and the distance function seems to lose its discriminative power. This phenomenon is called the concentration of distances.

Let $\mathcal{X}=\left\{x_{1}, x_{2}, \ldots, x_{N}\right\} \subset \mathbb{R}^{m}$ be a set of $N$ data points from the $m$-dimensional Euclidean space. Let $q \in \mathbb{R}^{m}$ be 


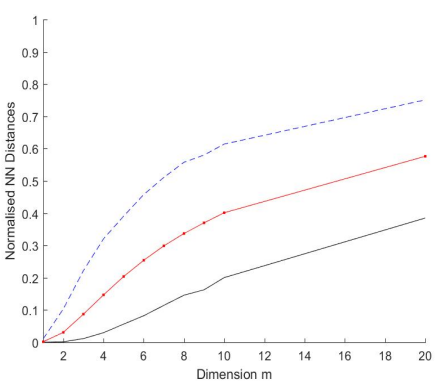

(a) $N=1000, m=1, \ldots, 20$

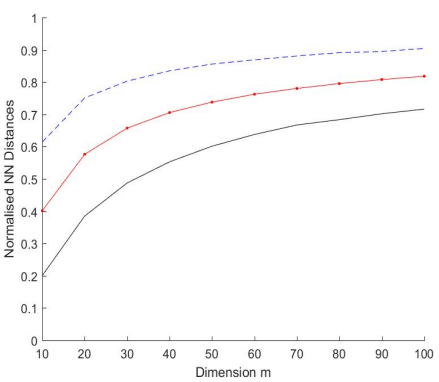

(b) $N=1000, m=10, \ldots, 100$

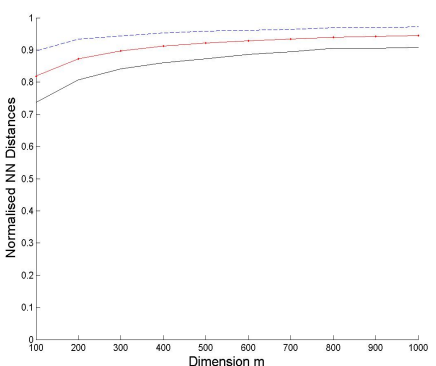

(c) $N=1000, m=100, \ldots, 1000$

Fig. 1. Concentration exhibited by the Euclidean distance when moving from low to high dimensions $-k_{M}(--), k_{m}(-), k_{A}(-\cdot-)$

an arbitrary but fixed query point and consider a distance function $\rho$ to calculate the distances between points in $\mathcal{X}$ for instance, $\rho$ could be the Euclidean distance (see (2) in Section 2). Let $x^{-}$and $x^{+}$be the nearest and farthest points to $q$, i.e., $x^{-}=\arg \min _{x_{i} \in X} \rho\left(x_{i}, q\right), \quad x^{+}=$ $\arg \max _{x_{i} \in X} \rho\left(x_{i}, q\right)$. As the dimension $m \rightarrow \infty$, one finds that $\rho\left(q, x^{-}\right) \approx \rho\left(q, x^{+}\right)$, which means that the distance of a query to the farthest point approaches the distance of the query to its nearest point. Since $\rho\left(q, x^{-}\right) \leq \rho\left(q, x_{i}\right) \leq$ $\rho\left(q, x^{+}\right)$for $1 \leq i \leq N$, all distances to $q$ begin to concentrate and are confined to a small domain. In other words, we can say that all the points in $\mathcal{X}$ are almost at the same distance to $q$. Thus the distances become less discriminative as the dimension grows and the distances between any two points begin to converge.

\subsection{An Empiricial Illustration}

Let us compare the Nearest Neighbour (NN) distances of an arbitrary query point with the average of pairwise distances of a given data set. Let $\mathcal{X}=\left\{x_{i}\right\}_{i=1}^{N}$ be $N$ data points. Let $Y_{\max }, Y_{\min }, Y_{\text {avg }}$ denote the maximum, minimum and average of the Nearest Neighbour Euclidean distances. For instance, if $z_{i}$ denotes the NN distance of $x_{i}$ for each $i=1, \ldots, N$, then $Y_{\max }=\max \left\{z_{i}: 1 \leq i \leq N\right\}$. We calculate $Y_{\min }, Y_{\text {avg }}$, similarly. Let $Y_{\mathcal{X}}$ denote the average of all pairwise distances in $\mathcal{X}$.

Let $k_{M}=\frac{Y_{\max }}{Y_{\mathcal{X}}}$ denote the normalised maximum NN distance w.r.t. the average of all pairwise distances. Similarly, let $k_{m}$ and $k_{A}$ denote the normalised minimum and average NN distances w.r.t. the average of all pairwise distances.

In Figs. 1(a) - (c), we plot the above three indices for $N=1000$ data points generated from Uniform distribution, viz., $\mathcal{X} \sim \mathcal{U}\left([-1,1]^{m}\right)$, for varying dimensions, $m=1, \ldots, 1000$. The plots allow us to make the following observations:

- In low dimensions, we see that $k_{m} \ll 1$ and there is enough separation between $k_{m}$ and $k_{M}$, i.e., there is sufficient contrast present and hence points are well separated, see Fig. 1 (a).

- In medium dimensions, i.e., up to 100 dimensions, $0 \ll$ $k_{m}<k_{A}<k_{M}$, which means that the minimum NN distances are beginning to increase and one can already see the presence of $\mathrm{CoD}$, see Fig. 1 (b).

- However, as dimension increases, $k_{m} \rightarrow 1, k_{M} \sim k_{A}$ and $k_{m} \sim k_{A}$, i.e., the normalised maximum NN distances and the normalised minimum NN distances both converge to the normalised average NN distances. There is not much contrast present between the distances, i.e., all the distances seem to concentrate around the average value of the pairwise distances. Thus all points become almost equidistant to each other, see Fig. 1 (c).

- A 3D surface plots of these indices for different values of $m$ and $N$, see Fig. 2, shows that increasing $N$ does not change the observed trend.

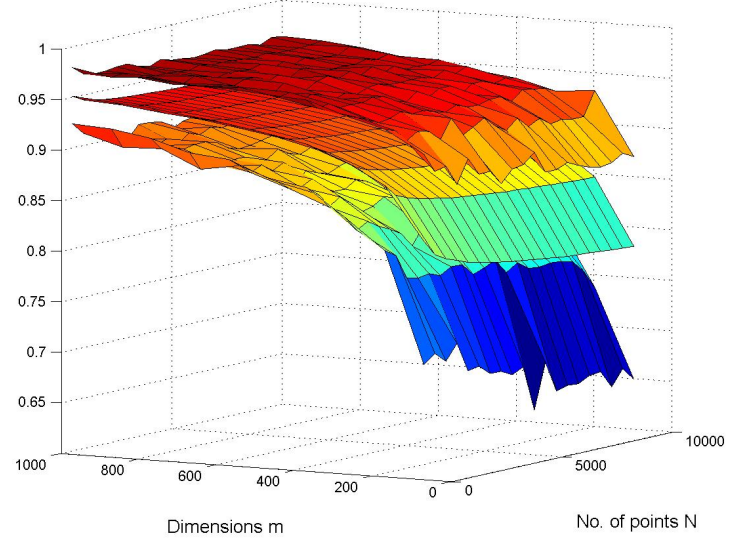

Fig. 2. Plots of the surfaces of $k_{m}$ (bottom most), $k_{A}, k_{M}$ (top most) for different values of $m$ and $N$.

\subsection{Why is CoD important?}

The concept of distance, or its dual notion of similarity, is all-pervasive and plays a central role in almost every algorithm or method in data analysis, from classification to clustering to similarity searches to pattern recognition. In many of these applications and algorithms, the distance functions which are useful in low dimensions are no longer effective in high dimensions, largely due to the affecting role of the $\mathrm{CoD}$ phenomenon. There are many domains 
where data are high dimensional and, thus, CoD poses an immediate and serious threat to their applicability to real world scenarios.

Let us consider searching, which is one of the most fundamental tasks used in every stream. The basic aim of similarity searching is to find an object or a set of objects similar to the given query object. For instance, in face recognition, one needs to search for a picture that is similar to the given query face in a database of images. A picture is made up of hundreds of thousands/millions of pixels and hence is a high dimensional object. Similarity searching methods, typically employ some kind of a distance function to measure the closeness between two objects. However, as shown above, due to the high dimensionality of the data, all pairwise distances can converge and hence our search might return a lot of candidates similar to our query object. This clearly puts a question mark on the usefulness of distance functions in high dimensions, see also [1], [18], [21].

Many nearest neighbour searching algorithms become computationally quite expensive in high dimensions [7], [6], [10], [16], [20], [32]. However, it is made even more difficult by $\mathrm{CoD}$. In fact, $\mathrm{CoD}$ raises the issue of whether or not the nearest neighbour is meaningful [9] in high dimension. Thus, in high dimensions, not only the efficiency of an algorithm is at stake, but also its effectiveness.

\subsection{Motivation for this work}

Since the seminal paper of Beyer et al. [9] on the concentration of distances, studies that deal with this phenomenon typically employ an index to illustrate their point. There exist 3 major indices that are often used in such works, viz., the relative contrast $\xi$, relative variance $\gamma$ and the concentration function $\alpha$. While all these indices are excellent illustrators of whether a distance function $\rho$ exhibits concentration or not, they do have their own merits and drawbacks. On the one hand, while both $\xi, \gamma$ are empirical indices, and are hence easy to calculate, the relative contrast $\xi$ is not amenable for theoretical analysis, while both $\xi, \gamma$ are not conducive to measure the level or rate of concentration. On the other hand, the concentration function $\alpha$ overcomes some of these drawbacks, but is extremely computationally intensive to calculate.

Given a data set $\mathcal{X}$ and a set of distance functions $\rho_{i}$, there does not exist any index, so far, that is able to compare them and suggest or indicate their suitability w.r.t. their level of concentration on $\mathcal{X}$. Thus there is a need for an efficient empirical index that orders a given set of distance functions w.r.t. their suitability, vis-á-vis their level of concentration. This forms the main motivation of this submission.

\subsection{Main Contributions of this work}

The main contributions of this work are twofold. Firstly, we propose a novel efficient and empirical index function, called the Dispersion Function $\lambda_{\rho}$, that not only illustrates whether a distance function $\rho$ exhibits concentration or not but also enables us to measure the rate at which it concentrates. This differs from the concentration function $\alpha_{\rho}$ in two aspects, viz.,

(i) $\lambda_{\rho}$ is far less computationally intensive than $\alpha_{\rho}$, (ii) $\lambda_{\rho}$ being an empirical measure can be calculated on any given data set $\mathcal{X}$. Further, $\lambda_{\rho}$ only makes use of the internal characteristics of the given data set $\mathcal{X}$ and hence, unlike $\alpha_{\rho}, \lambda_{\rho}$ can be calculated even if the underlying distribution of $\mathcal{X}$ is unknown.

Secondly, based on the dispersion function $\lambda_{\rho}$, we have proposed an index $\tau_{\rho}$ which enables us to compare distances and indicate their suitability w.r.t. their level of concentration on $\mathcal{X}$.

\section{Indices to Illustrate and/or Measure CoD}

As discussed in Section 1, distances do tend to concentrate in higher dimensions. The illustration in Section 1.3 was done for Eucidean distances, i.e., distances calculated based on what is often referred to as an $\mathcal{L}_{2}$ distance, since it is a member of the family of Minkowski's $\mathcal{L}_{p}$-distances for $p \in[1, \infty)$, given as follows for a pair of vectors $x=\left(x_{1}, x_{2}, \ldots, x_{m}\right), y=\left(y_{1}, y_{2}, \ldots, y_{m}\right) \in \mathbb{R}^{m}$ :

$$
\begin{gathered}
\|x\|_{p}=\left(\sum_{i=1}^{m}\left|x_{i}\right|^{p}\right)^{\frac{1}{p}}, \\
\mathcal{L}_{p}(x, y)=\|x-y\|_{p}=\left(\sum_{i=1}^{m}\left|x_{i}-y_{i}\right|^{p}\right)^{\frac{1}{p}} .
\end{gathered}
$$

Note that $\mathcal{L}_{p}$ is a metric [11].

It can be easily shown that the above indices, viz., $k_{M}, k_{m}, k_{A}$, behave similarly when we use a different $\mathcal{L}_{p^{-}}$ distance, i.e., they all still do converge. However, the following questions arise:

(i) Even if all $\mathcal{L}_{p}$-distances concentrate, do they all concentrate in the same manner? In which case, can one talk about the rate of concentration?

(ii) Are there indices that allow comparison between different distance functions w.r.t. their concentration? Are they calculable empirically? Are they also amenable for theoretical studies?

Since the seminal paper on this topic by Beyer et al. [9], there have been many studies dealing with the above posers. In this section, we give a brief yet substantive review of these works and the indices proposed therein, highlight their advantages and indicate the contexts in which they are not readily applicable, thus leading up to the motivation behind this work.

\subsection{Fixing the notation}

We introduce some notations and concepts which will be used in the rest of the paper.

- The triple $(\Omega, \rho, \mu)$ will denote a measurable metric space, where $\Omega$ is the domain, $\rho$ is the metric on $\Omega$ and $\mu$ is a probability measure on $\Omega$.

- Further, the measure $\mu$ we consider will always be absolutely continuous and hence we can associate a distribution $\mathcal{R}$ which will be used to obtain a finite sample of $N$-points $\mathcal{X}=\left\{x_{1}, x_{2}, \ldots, x_{N}\right\} \subset \Omega$. We will then write $\mathcal{X} \sim \mathcal{R}$ to denote that the data set $\mathcal{X} \subset \Omega$ has been generated using the distribution $\mathcal{R}$. Often the quadruple $(\Omega, \mathcal{X}, \rho, \mu)$ is termed as a Similarity Workload, see [25], $[26]$. 
- We assume that there always exist a $\mathbf{0} \in \Omega$ designated as the origin of the domain $\Omega$. This is almost always true since usually $\Omega \subseteq \mathbb{R}$.

- $\mu^{*}$ will denote the counting measure, i.e., if $\mathcal{X}$ is finite, $\mu^{*}(\mathcal{X})=\sharp \mathcal{X}$, the cardinality of $\mathcal{X}$.

- Note that if $\Omega \subseteq \mathbb{R}^{m}$, then we will also use the notation $\Omega^{m}, \mathcal{X}^{m}$ for added emphasis. In such cases, the other quantities like $\mu^{m}, \rho^{m}, \mathcal{R}^{m}$, etc., are appropriately defined.

- By $\|\cdot\|$ we denote a real valued function on $\Omega$, i.e, $\|\cdot\|$ : $\Omega \rightarrow \mathbb{R}$, which is taken to measure the distance of an $x \in \Omega$ to the origin $\mathbf{0} \in \Omega$, i.e., $\|x\|=\rho(x, \mathbf{0})$. To remain consistent with earlier works, we term $\|x\|$ to be the norm of the vector $x$, even when $\|\cdot\|$ does not satisfy all the properties of a norm, as is common in the literature.

- $D_{\max }^{m}\left(D_{\min }^{m}\right)$ denotes the maximum (minimum, resp.) of the norms in a given data set $\mathcal{X}^{m}$ of $N$ points, i.e., the distance of the farthest (nearest, resp.) point in $\mathcal{X}^{m}$ to the origin w.r.t. the metric $\rho$ :

$$
\begin{gathered}
D_{\max }^{m}=\max \left\{\left\|x_{i}^{m}\right\|=\rho\left(x_{i}^{m}, \mathbf{0}\right): x_{i}^{m} \in \mathcal{X}^{m}\right\}, \\
D_{\min }^{m}=\min \left\{\left\|x_{i}^{m}\right\|=\rho\left(x_{i}^{m}, \mathbf{0}\right): x_{i}^{m} \in \mathcal{X}^{m}\right\} .
\end{gathered}
$$

- $E[Z]$ and $\operatorname{var}[Z]$ will denote the expectation and variance of a random variable $Z$.

\subsection{Existence of CoD: Theoretical Analysis}

Towards discussing the questions raised in Section 2 above, we begin by recalling the seminal result of Beyer et al. [9], wherein they discussed the existence of meaningful nearest neighbours in high dimension. Their result showed that under some reasonable assumptions on the data distribution $\mathcal{R}$, distance functions do concentrate.

Theorem 2.1 ( [9], Theorem 1). Let $\left(\Omega^{m}, \rho^{m}, \mu^{m}\right)$ be an $m$-dimensional measurable metric space, let $\mathcal{X}^{m}=$ $\left\{x_{1}^{m}, x_{2}^{m}, \ldots, x_{N}^{m}\right\}$ be a finite sample of $N$ points such that $x^{m} \sim \mathcal{R}^{m}$ and $D_{\text {max }}^{m}, D_{\text {min }}^{m}$ are as defined above. Further, let $E\left[\left\|x^{m}\right\|\right]$ and $\left.\operatorname{var}\left[\| x^{m}\right) \|\right]$ be finite and $E\left[\| x^{m}||\right] \neq 0$. If

$$
\lim _{m \rightarrow \infty} \operatorname{var}\left(\frac{\left\|x^{m}\right\|}{E\left\|x^{m}\right\|}\right)=0,
$$

then for all $\varepsilon>0$,

$$
\lim _{m \rightarrow \infty} P\left[D_{\max }^{m} \leq(1+\varepsilon) D_{\min }^{m}\right]=1 .
$$

The above result points out that nearest neighbor searching is not meaningful when the variance of the ratio of the distance between any two random points, drawn from the data distribution, and the expected distance between them converges to zero as dimension goes to infinity. This, in essence, means that almost all points are equidistant to the query point.

Theorem 2.1 clearly discusses only a sufficient condition for concentration, i.e., the distance to the nearest neighbor and the distance to the farthest neighbor tend to converge, in a probabilistic sense, as the dimension $m$ increases. In other words, we get a poor contrast if the spread between the points tends towards 0 . However, the question of whether this condition is also necessary was not known. Almost after a decade after the work of Beyer et al., the converse of Theorem 2.1 was proved by Durrant and Kabán, see [12], Theorem 2, pg. 387.
TABLE 1

Some Important Notations

\begin{tabular}{|c|l|}
\hline$\Omega$ & Non-empty Domain \\
\hline$\rho$ & Distance Function \\
\hline $\mathcal{X} \subset \Omega$ & Data set \\
\hline$\xi_{\rho}$ & Relative Contrast w.r.t. $\rho$ \\
\hline$\gamma_{\rho}$ & Relative Variance w.r.t. $\rho$ \\
\hline$\alpha_{\rho}$ & Concentration Function w.r.t. $\rho$ \\
\hline$\lambda_{\rho}$ & Dispersion Function w.r.t. $\rho$ \\
\hline $\mathcal{L}_{p}$ & $p$-th Minkowski norm $p \geq 1$ \\
\hline $\mathcal{F}_{p}$ & Fractional norm $-\mathcal{L}_{p}$ with $p \in(0,1)$ \\
\hline$\mu^{*}$ & Counting Measure \\
\hline$|\mathcal{X}|$ & Cardinality of the dataset $\mathcal{X}$ \\
\hline$\mu_{\mathcal{X}}^{*}$ & Normalised Counting Measure w.r.t. $|\mathcal{X}|$ \\
\hline
\end{tabular}

\subsection{Some Indices to Illustrate CoD}

Based on the theoretical results of Beyer et. al., [9] proving the existence of $\mathrm{CoD}$ in high dimensions, two indices have been proposed to study the tendency of concentration among different distances.

\subsubsection{Relative Contrast - An Index to Illustrate CoD}

The first of them is the Relative Contrast proposed by Aggarwal et al. [3].

Definition 2.2 ([3], Pg. 422). Let us consider an $m$ dimensional similarity workload, $\left(\Omega^{m}, \mathcal{X}^{m}, \rho^{m}, \mu^{m}\right)$. The Relative Contrast (RC), w.r.t. $\rho$, is defined as

$$
\xi_{\rho}(m)=\frac{D_{\max }^{m}-D_{\min }^{m}}{D_{\min }^{m}} .
$$

Defining relative contrast thus, Aggarwal et al. [3] showed that when $\rho$ is any of the Minkowski norms $\mathcal{L}_{p}$ for $p \in[1, \infty), \xi_{\mathcal{L}_{p}}(m) \rightarrow 0$ as $m \rightarrow \infty$. Interestingly, based on the bounds obtained for $\xi_{\mathcal{L}_{p}}(m)$ they argued that if the exponent $p \in(0,1)$ in $(1)$ then such $p$-norms, which they called fractional norms and were denoted by $\mathcal{F}_{p}$, were better than Minkowski distances $\mathcal{L}_{p}$. It should be mentioned that when $p \in(0,1)$ then the fractional distances $\mathcal{F}_{p}$ are not norms, or even a metric, since they do not satisfy the triangle inequality. Thus in the sequel, we refer to all such functions with the more general term distance functions.

\subsubsection{Relative Variance - Another Index to Illustrate CoD}

While Aggarwal et al. [3] took their motivation from (4) of Theorem 2.1 to propose $\xi_{\rho}$, François et al. [15] proposed yet another index, but this time taking their cue from (3) of Theorem 2.1, to demonstrate if a distance function suffers from the concentration phenomenon or not.

Definition 2.3 ([15], pg. 877). Given an $m$ - dimensional similarity workload, $\left(\Omega^{m}, \mathcal{X}^{m}, \rho^{m}, \mu^{m}\right)$, the relative variance of the distance function $\rho$ is defined as:

$$
\gamma_{\rho}(m)=\frac{\sqrt{\operatorname{Var}\left(\left\|x^{m}\right\|\right)}}{E\left(\left\|x^{m}\right\|\right)},
$$

where, as usual, $\|x\|=\rho(x, \mathbf{0})$.

The relative variance $\gamma_{\rho}$ illustrates the concentration of distances by comparing the spread with the expectation of the distances. If $\gamma_{\rho}$ has a small value then it indicates that distances are concentrated and a large value for $\gamma_{\rho}$ denotes a good amount of spread between the distances. In some 
sense it is similar to relative contrast, as $\xi_{\rho}$ also compares the measure of spread to the measure of location.

In fact, Theorem 2.1 and its converse can be restated as follows based on the above indices: If the relative variance is not tending to zero then the relative contrast will also not converge to zero and therefore one does obtain a good separation between points.

Remark 2.4. Following are some of the merits and demerits of the indices $\xi_{\rho}$ and $\gamma_{\rho}$ :

$\oplus$ It does illustrate the concentration of distances well. Fig. 3(a) plots the relative contrast of the Euclidean distance $\mathcal{L}_{2}$ on the set $\mathcal{X}$ consisting of $N$ data points, where $\mathcal{X} \sim \mathcal{U}\left([-1,1]^{m}\right)$ and $N=100 m$. It is clear from the plots that in low dimensions, viz., $m=1, \ldots, 10, \xi_{\mathcal{L}_{2}}$ is quite high, while even in medium dimensions, viz., $m=10, \ldots, 100, \xi_{\mathcal{L}_{2}}$ starts to fall drastically and in high dimensions, viz., $m=100, \ldots, 1000, \xi_{\mathcal{L}_{2}}$ is almost zero. $\gamma_{\rho}$, like $\xi_{\rho}$, does illustrate the concentration of distances well. Once again, Fig. 3(b) illustrates that in low dimensions $\gamma_{\mathcal{L}_{2}}$ is far away from zero, but in medium to large dimensions $\gamma_{\mathcal{L}_{2}}$ starts to approach zero faster.

$\oplus$ Both $\xi_{\rho}$ and $\gamma_{\rho}$ are empirically calculable and hence are applicable on any distance function.

$\ominus \xi_{\rho}$ is not amenable for theoretical analysis, as finding the distributions of minimum and maximum pairwise distances in a data set with given probability distribution for most distances is extremely complicated. Even in the case of $\mathcal{L}_{p}$ and $\mathcal{F}_{p}$ norms only some loose bounds have been obtained and when $N$ is finite. Where $\gamma_{\rho}$ overtakes $\xi_{\rho}$ is in its amenability for theoretical analysis, as is clearly demonstrated by François et al. [15]. Note, however, that theoretical analysis can become difficult with arbitrary distance functions.

$\ominus$ Both $\xi_{\rho}$ and $\gamma_{\rho}$ illustrate the concentration of a particular distance in the asymptotic case as $m \rightarrow \infty$. However, given two distances, say $\rho_{1}, \rho_{2}$, and a specific data set $\mathcal{X}^{m}$ (thus the dimensionality $m$ is fixed), it is not clear if the values $\xi_{\rho_{1}}(m)$ and $\xi_{\rho_{2}}(m)$ allow us to compare the distances $\rho_{1}, \rho_{2}$ vis-á-vis their concentration. Note that $\xi_{\rho}, \gamma_{\rho}$ are not strictly decreasing functions of $m$.

\subsection{A theoretical Index to Measure CoD}

While $\xi_{\rho}$ and $\gamma_{\rho}$ illustrate the concentration phenomenon well, they do not give any information on the rate at which a distance function concentrates. Recent studies, see Pestov [25], have started considering a more general mathematical function to measure concentration.

Definition 2.5 (cf. [37], [24], [25]). Let us be given a measurable metric space $(\Omega, \rho, \mu)$. The concentration function $\alpha_{\rho}: \mathbb{R}^{\geq 0} \rightarrow\left[0, \frac{1}{2}\right]$ is defined as follows:

$\alpha_{\rho}(\varepsilon)= \begin{cases}1-\inf \left\{\mu\left(A_{\varepsilon}\right): A \subseteq \Omega \& \mu(A) \geq 1 / 2\right\}, & \varepsilon>0, \\ \frac{1}{2}, & \varepsilon=0,\end{cases}$ where $A_{\varepsilon}=\{x \in \Omega: \rho(x, a)<\varepsilon$ for some $a \in A\}$.

The value $\alpha_{\rho}(\varepsilon)$ gives an upper bound on the measure of the complement to the $\varepsilon$-neighborhood $A_{\varepsilon}$ of every subset
$A$ of measure greater than or equal to $\frac{1}{2}$. It can be easily seen that $\alpha_{\rho}$ is a decreasing function. Thus, the rate of concentration of a distance function $\rho$, in the considered workload, is measured based on the rate at which $\alpha_{\rho}$ decreases.

If a distance function $\rho$ concentrates, the concentration function $\alpha_{\rho}$ approaches zero faster. The smaller the value of $\varepsilon$ at which $\alpha_{\rho}(\varepsilon)=0$, the faster the distance function concentrates. In fact, the rate at which $\alpha_{\rho}$ decreases is illustrative of the fact that the pairwise distances, as measured by $\rho$, concentrate near their mean/median value.

In Example 2.6, we consider some simple measurable metric spaces $(\Omega, \rho, \mu)$ and plot their respective concentration functions in Fig. 3(c), which shows that $\alpha_{\rho}$ does measure the rate of concentration, i.e., how fast a given distance $\rho$ concentrates in a domain of interest $\Omega$ with respect to the data distribution obtained from the measure $\mu$.

Example 2.6.

(i) Let us consider the space $\left(\Omega_{1}, \rho, \mu\right)$, where $\Omega_{1}=$ $[0,1] \cup[2,3], \rho$ is the usual metric on $\mathbb{R}$, viz., the $\mathcal{L}_{1}$ metric and $\mu$ is the Lebesgue measure. The corresponding concentration function $\alpha_{\mathcal{L}_{1}}^{\Omega_{1}}(-\bullet-)$ is plotted in Fig. 3(c).

(ii) Let us now consider the domains $\Omega_{2}=[0,1] \cup[1.1,2.1]$ and $\Omega_{3}=[-0.6,-0.1] \cup[0,1] \cup[1.1,1.6]$, while $\rho, \mu$ remain the same. The corresponding concentration functions $\alpha_{\mathcal{L}_{1}}^{\Omega_{2}}(-*-)$ and

$\alpha_{\mathcal{L}_{1}}^{\Omega_{3}}(-+-)$, respectively, are plotted in Fig. 3(c).

Following are some of the merits and demerits of the concentration function $\alpha_{\rho}$ :

$\oplus$ Not only does $\alpha$ illustrate the concentration of distances, it also allows us to measure it. In Example 2.6, we saw that the $\mathcal{L}_{1}$ distance behaves differently for different domains, even though the measure of the underlying domains $\mu\left(\Omega_{i}\right)$ was the same.

$\oplus$ The concentration function has been used in the analysis of many a measurable metric space to obtain theoretical bounds, see, for e.g., the monograph of Ledoux [24].

$\oplus$ Given a measurable space, $(\Omega, \mu)$ of an arbitrary but fixed dimension $m$, one can visually compare different distance functions $\rho_{i}$ based on the rate at which the corresponding $\alpha_{\rho_{i}}$ tends to zero.

$\ominus$ However, given two distances, say $\rho_{1}, \rho_{2}$, it is not clear if the functions $\alpha_{\rho_{1}}$ and $\alpha_{\rho_{2}}$ can be strictly ordered, based on the usual point-wise ordering of functions. Thus even if $\alpha_{\rho_{1}}(\varepsilon)<\alpha_{\rho_{2}}(\varepsilon)$, there can exist an $\varepsilon^{\prime} \neq \varepsilon$ such that $\alpha_{\rho_{1}}\left(\varepsilon^{\prime}\right)>\alpha_{\rho_{2}}\left(\varepsilon^{\prime}\right)$. Thus other than the visual cues, purely based on $\alpha_{\rho_{i}}$ 's it is not apparent how to compare distances w.r.t. their concentration.

$\ominus$ Further, if we do not know the underlying distribution of a particular dataset a priori, i.e., if $\mu$ is unknown, we cannot determine $\alpha_{\rho}$ theoretically.

$\ominus$ While it is amenable for certain kind of theoretical analysis, as an empirical index $\alpha_{\rho}$ is highly computationally expensive. For large sets calculating $\alpha_{\rho}$ is very cumbersome as we need to find every subset of $\Omega$ with measure at least half. In fact, using the counting measure $\mu_{\mathcal{X}}^{*}$, given a set with cardinality $N$, 


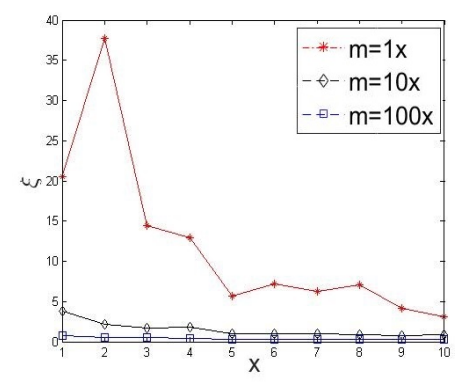

(a) $\xi_{\mathcal{L}_{2}}, N=100 m$

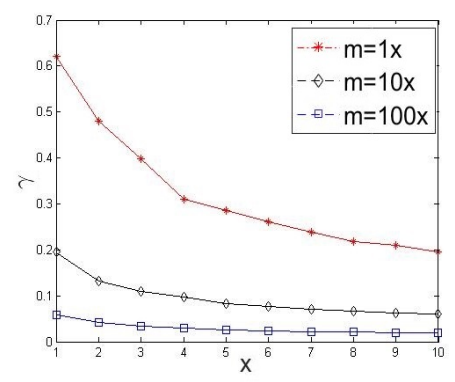

(b) $\gamma_{\mathcal{L}_{2}}, N=100 \mathrm{~m}$

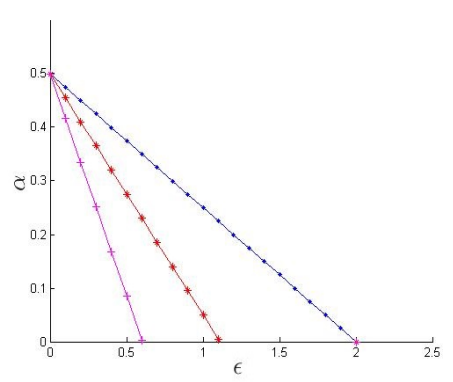

(c) $\alpha_{\mathcal{L}_{1}}^{\Omega_{i}}$

Fig. 3. (a) Relative Contrast and (b) Relative Variance of the Euclidean distances as dimensions increase, see Remark 2.4. (c) Concentration functions $\alpha_{\mathcal{L}_{1}}^{\Omega_{1}}(-\bullet-), \alpha_{\mathcal{L}_{1}}^{\Omega_{2}}(-*-), \alpha_{\mathcal{L}_{1}}^{\Omega_{3}}(-+-)$ of Example 2.6.

TABLE 2

Comparison between the indices $\xi_{\rho}, \gamma_{\rho}$ and $\alpha_{\rho}:(\mathrm{EC})$ - Empirical Calculations, (TA) - Theoretical Analysis, (MC) - Measuring Concentration, $(\mathrm{CD})$ - Comparing Distances.

\begin{tabular}{|c|c|c|c|c|}
\hline \multirow{2}{*}{ Index } & \multicolumn{4}{|c|}{ Suitable for } \\
\cline { 2 - 5 } & (EC) & (TA) & (MC) & (CD) \\
\hline$\xi_{\rho}$ & $\checkmark$ & $\times$ & $\times$ & $\times$ \\
\hline$\gamma_{\rho}$ & $\checkmark$ & $\checkmark$ & $\times$ & $\times$ \\
\hline$\alpha_{\rho}$ & $\times$ & $\checkmark$ & $\checkmark$ & $\times$ \\
\hline
\end{tabular}

the number of subsets with measure greater than $\frac{1}{2}$ is equal to $\sum_{k=\frac{N}{2}}^{N}{ }^{N} C_{k}=2^{N-1}$.

2.5 Motivation for this work - Need for an Efficient Empirical Index to Measure CoD

It is clear from the discussions so far that, on the one hand, $\xi_{\rho}, \gamma_{\rho}$ are best suited in empirical settings and are efficiently calculable, but $\alpha_{\rho}$ does not enjoy these properties. On the other hand, $\alpha_{\rho}$ can be useful in comparing distances w.r.t. their concentration, while neither $\xi_{\rho}$ nor $\gamma_{\rho}$ gives us that information. Table 2 summarises the properties of the above indices against these parameters, from whence we see the need for an efficient empirical index, á la $\xi_{\rho}, \gamma_{\rho}$, and that which would measure the concentration and hence allow us to compare between different distance functions, á la $\alpha_{\rho}$. Our approach towards defining this index stems from the concept of stability of queries. In the next sections, we discuss these in detail and come up with an empirical index that upper bounds $\alpha_{\rho}$, which is also comparatively easier to calculate than $\alpha_{\rho}$.

\section{A Novel Efficient Empirical Index to Measure CoD}

In this section, we recall the concept of stability of range queries and discuss the stability of workloads. Based on these discussions, we present our novel index that not only illustrates and measures concentration, but with the help of which we will also be able to compare distances vis-á-vis their concentration.

\subsection{Stability of a Query}

Let $(\Omega, \mathcal{X}, \rho, \mu)$ be a given similarity workload. Let a query $q \in \Omega$ and an $\varepsilon \in \overline{\mathbb{R}}_{+}=[0, \infty)$ be given. By a rangequery problem we refer to the determination of the set of all points in $\mathcal{X}$ that are within $\varepsilon$ units away from $q$, i.e., we need to find the $\varepsilon$-neighbourhood of $q$ in $\mathcal{X}$ :

$$
S=\mathrm{N}(q, \varepsilon)=\left\{x^{\prime} \in \mathcal{X}: \rho\left(x^{\prime}, q\right) \leq \varepsilon\right\} .
$$

In [9], the authors discuss when a range-query is stable by defining the stability of a range-query as follows:

Definition 3.1 ([25], Pg. 48, cf. [9], Definition 1). Given a query point $q \in \Omega$ and an $\varepsilon \in \overline{\mathbb{R}}_{+}$, a range-query is said to be $\varepsilon$-unstable if

$$
\mu_{\mathcal{X}}^{*}(\mathrm{~N}(q,(1+\epsilon) * \delta)) \geq \frac{\mu_{\mathcal{X}}^{*}(X)}{2},
$$

where, $\delta=\min \{\rho(q, x): x \in \mathcal{X}\}$, the NN-distance of $q$.

In other words, as formulated initially in [9], a range-query is said to be unstable if most of the data set is covered within the $\varepsilon-\delta$ sphere of the query $q$. The subsequent quantification to half (from most) of the data set in Definition 3.1 was done by Pestov [25].

Taking a cue from Definition 3.1, we discuss the stability of a particular workload and propose an index that will help us in achieving our goals.

\subsection{The $g$ - $\delta$-Count}

Let $\mathbb{N}_{N}=\{1,2, \ldots, N\}$. Let $\mathcal{X}$ be a given data set whose cardinality is $N$, i.e., $\sharp \mathcal{X}=N$.

Consider an $x \in \mathcal{X}$ and let $\delta$ denote the NN distance of $x$. For a $g \in \overline{\mathbb{R}}_{+}$, let us define the $g$ - $\delta$ count of the point $x$ as

$$
C(x, g \delta)=\mu_{\mathcal{X}}^{*}(\mathrm{~N}(x, g \delta)) .
$$

Clearly, $C(x, g \delta)$ gives the fraction of the number of data points in the $g-\delta$ neighborhood of $x$. Note that $\mu_{\mathcal{X}}^{*}$ is the normalised counting measure, i.e., for an $A \subset X$ and $|X|<$ $\infty$ we have $\mu_{\mathcal{X}}^{*}(A)=\frac{|A|}{|X|}$.

For small values of $g$, if the $C(x, g \delta)$ values of most of the $x \in \mathcal{X}$ are high, then one surmises that more points lie in the dilated $g$ - $\delta$ neighborhood of each $x \in \mathcal{X}$ and hence the data are distributed very close to each other 
and the relative distances between the data points will be small. Thus, $C(\cdot, g \delta)$ does keep track of the concentration of points. Specifically, given a dataset, even without the information of the distribution of the dataset, $C(\cdot, g \delta)$ is computable and hence further analysis is possible.

Now, we define $C^{*}$, the complement of $C$ as follows:

$$
C^{*}(x, g \delta)=1-C(x, g \delta) .
$$

$C^{*}(x, g \delta)$ gives the fraction of the data set that the point $x$ is not able to arrest through its dilated $g-\delta$ neighborhood.

Clearly, if $C(x, g \delta)$ is large for a point $x$ then $C^{*}(x, g \delta)$ will be small. Thus, when $g$ is relatively small, small values of $C^{*}(\cdot, g \delta)$ for most of the data set would indicate that distances are concentrating and vice versa. Note, however, that the above observation is valid only if a large number of points have small values of $C^{*}(\cdot, g \delta)$. For instance, if $C^{*}(\cdot, g \delta)$ values are very large for only a minority of the data points, it does not mean that the distances are not concentrating. It may happen that these points are outliers and rest of the data points that are not captured by these outliers are closely packed. Therefore, we need to check the overall behavior of all the data points. Taking cue from this observation, we propose a novel index that will help us to accomplish our objective in the next section.

\subsection{The Dispersion Function - $\lambda_{\rho}$}

Consider the similarity workload $\left(\Omega, \mathcal{X}, \rho, \mu_{\mathcal{X}}^{*}\right)$. If $\delta_{i}$ is the NN distance of an $x_{i} \in \mathcal{X}$, let $\delta_{0}$ denote the maximum of the $\mathrm{NN}$ distances in $\mathcal{X}$, i.e.,

$$
\delta_{0}=\max _{x_{i} \in \mathcal{X}}\left\{\delta_{i}\right\}=\max _{x_{i} \in \mathcal{X}}\left\{\min _{x_{j} \in \mathcal{X}} \rho\left(x_{i}, x_{j}\right)\right\} .
$$

Definition 3.2. Let us consider the similarity workload $\left(\Omega, \mathcal{X}, \mu_{\mathcal{X}}^{*}, \rho\right)$ and let $\delta_{0}$ be as defined in (5). The dispersion function $\lambda_{\rho}:[-1, \infty) \rightarrow[0,1]$ is defined as follows:

$$
\lambda_{\rho}(\varepsilon)=\underset{x_{i} \in \mathcal{X}}{\operatorname{avg}}\left\{C^{*}\left(x_{i},(1+\varepsilon) \delta_{0}\right)\right\},
$$

where avg is the the usual statistical average of the values.

What does the dispersion function $\lambda_{\rho}$ really indicate? For a given $\varepsilon>0, \lambda_{\rho}$ returns the average of the fraction of the data set that is not captured by a data point in its dilated $(1+\varepsilon) \delta_{0}$ neighborhood. Thus, when $N$ is large, high values of $\lambda_{\rho}$ indicate that a large part of the data set are such that most of the data are lying at a distance greater than $(1+\varepsilon) \delta_{0}$ to each of them. If we take $\varepsilon$ to be small, data are at least $\delta_{0}$ distance away and so data will still be well separated. Thus, essentially, $\lambda_{\rho}$ can be considered as a statistical measure of the dispersion as measured by the distance function $\rho$. As will be shown in the next section, where we discuss its properties, the dispersion function $\lambda_{\rho}$ does have many desirable properties, one of which is that it forms an upper bound for the concentration function $\alpha_{\rho}$ when the distance function under consideration is known to concentrate; thus if $\lambda_{\rho}$ decreases at a faster rate, then so does $\alpha_{\rho}$.

From the definition of $\lambda_{\rho}$ and the discussion above, the following remarks are readily verifiable:
TABLE 3

Real Data sets

\begin{tabular}{|l|c|c|}
\hline Dataset $\mathcal{X}_{i}$ & Dimension $m$ & $\sharp$ of datapoints $N$ \\
\hline Splice & 60 & 1000 \\
Protein & 357 & 6621 \\
Colon Cancer & 2000 & 62 \\
Gisette & 5000 & 6000 \\
Duke & 7129 & 44 \\
Dexter & 20000 & 300 \\
\hline
\end{tabular}

$\oplus \lambda_{\rho}$ is an empirical function and illustrates the concentration of distances well.

$\oplus \lambda_{\rho}$ is calculable for arbitrary distances.

$\oplus \lambda_{\rho}$, like $\alpha_{\rho}$, is calculable for any data set with fixed dimensionality.

$\oplus$ Even if we do not know the underlying distribution of a particular dataset a priori, i.e., even if $\mu$ is unknown, we can still determine $\lambda_{\rho}$.

However, it is not immediately clear whether $\lambda_{\rho}$, like $\alpha_{\rho}$, measures the rate of concentration or allows us to compare distance functions w.r.t. their concentration. We take this up in detail in the next section.

\section{Empirical and Theoretical Analysis of $\lambda_{\rho}$}

In this section, we discuss the properties and characteristics of the dispersion function $\lambda_{\rho}$. We begin by empirically plotting $\lambda_{\rho}$ for different distance functions $\rho$, on both synthetic and some real data sets and make some evidential observations based on them, some of which are also validated theoretically later on. Following this, we discuss the behaviour of the dispersion function on distance functions and show how $\lambda_{\rho}$ can help in distinguishing distance functions based on their concentration. Finally, we do a comparative study between $\lambda_{\rho}$ and $\alpha_{\rho}$.

\subsection{Studies on Synthetic and Real Datasets}

Let $\Omega=[-1,1]^{m}$ be the $m$-dimensional unit hyper cube. We consider two data sets $\mathcal{X}_{1}, \mathcal{X}_{2}$ of cardinality $N$, where

- $\mathcal{X}_{1}$ is a set of $N$ uniformly distributed points in $\Omega$, i.e., $\mathcal{X}_{1} \sim \mathcal{U}\left([-1,1]^{m}\right)$

- $\mathcal{X}_{2}$ is a set of $N$ points generated using a normal distribution with mean 0 and variance 0.09 , on each of the $m$ dimensions, so as to ensure that $\mathcal{X}_{2} \subset \Omega$, i.e., $\mathcal{X}_{2} \sim \mathcal{N}(0,0.3)$.

For the distance function $\rho$, we consider the Minkowski distance functions $\mathcal{L}_{p}$ for $p=1,2,3, \infty$ and the fractional distances $\mathcal{F}_{p}$ for $p=0.04,0.25$. We consider the following two sets of synthetic workloads $\mathcal{W}_{1}=\left(\Omega, \mathcal{X}_{1}, \rho_{i}\right)$ and $\mathcal{W}_{2}=\left(\Omega, \mathcal{X}_{2}, \rho_{i}\right)$, where $\rho_{i}$ is one of the 6 distance functions listed above. We typically took the number of data points $N=10 K$, restricted largely due to the computational power available, and plotted the $\lambda_{\rho_{i}}$ values for $m=10,100$ and 1000. In Figs. 4 (a)-(c) we plot the graph of different $\lambda_{\rho_{i}}$ as $\varepsilon$ varies from -1 to 3 in steps of 0.1 .

One of the main advantages of $\lambda_{\rho}$ over $\alpha_{\rho}$ is that it could be applied to real data sets, where usually there is no a priori knowledge about the underlying distribution. We consider the UCI data sets given in Table 3, i.e., the sets of workloads $\left(\mathcal{X}_{i}, \rho_{j}, \mu_{\mathcal{X}}^{*}\right)$, where $\rho_{j}, j=1,2, \ldots, 6$ are 


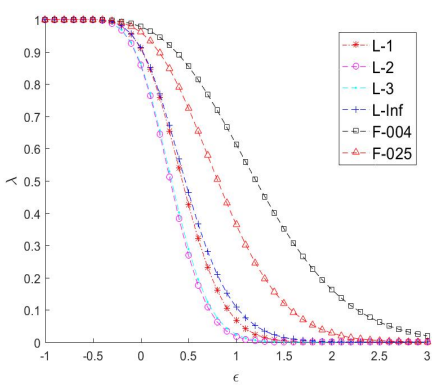

(a) Gaussian, $N=10 \mathrm{~K}, m=10$

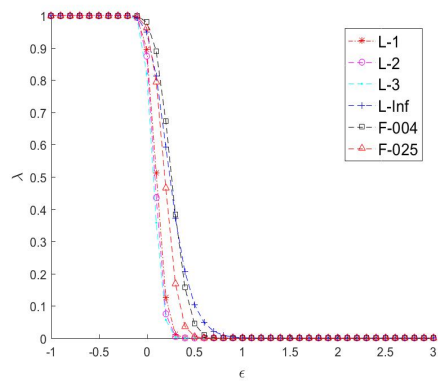

(b) Gaussian, $N=10 \mathrm{~K}, m=100$

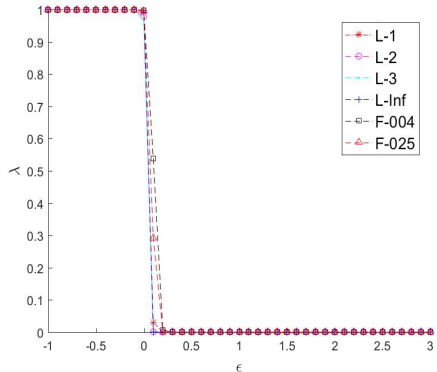

(c) Uniform, $N=10 \mathrm{~K}, m=1000$

Fig. 4. Plot of $\lambda_{\rho_{i}}$ for different workloads - see Section 4.1.

the same set of 6 Minkowski distance functions considered above. The superimposed plots of $\lambda_{\rho_{j}}$ for four of the workloads, with $m \geq 2000$, is given in Figs. 5 (a)-(d).

From Figs. 4 (a) - (c) and Figs. $5(\mathrm{a})-(\mathrm{d})$ we see that $\lambda_{\rho}$ is a decreasing function of $\varepsilon$. The following result demonstrates theoretically the above empirical observation.

Theorem 4.1. Given a similarity workload $(\Omega, \mathcal{X}, \rho, \mu), \lambda_{\rho}$ is a decreasing function, i.e., $\varepsilon_{1} \leq \varepsilon_{2} \longrightarrow \lambda_{\rho}\left(\varepsilon_{1}\right) \geq \lambda_{\rho}\left(\varepsilon_{2}\right)$.

Proof: Let $\varepsilon_{1} \leq \varepsilon_{2}$ for $\varepsilon_{1}, \varepsilon_{2} \in[-1, \infty)$ and $\delta_{0}>0$ be as defined in (5). Then,

$$
\begin{aligned}
& \left(1+\varepsilon_{1}\right) \delta_{0} \leq\left(1+\varepsilon_{2}\right) \delta_{0} \\
\Longrightarrow & \mathrm{N}\left(x_{i},\left(1+\varepsilon_{1}\right) \delta_{0}\right) \subset \mathrm{N}\left(x_{i},\left(1+\varepsilon_{2}\right) \delta_{0}\right) \quad(\forall i) \\
\Longrightarrow & \mu_{\mathcal{X}}^{*}\left(\mathrm{~N}\left(x_{i},\left(1+\varepsilon_{1}\right) \delta_{0}\right)\right) \leq \mu_{\mathcal{X}}^{*}\left(\mathrm{~N}\left(x_{i},\left(1+\varepsilon_{2}\right) \delta_{0}\right)\right)(\forall i) \\
\Longrightarrow & C\left(x_{i},\left(1+\varepsilon_{1}\right) \delta_{0}\right) \leq C\left(x_{i},\left(1+\varepsilon_{2}\right) \delta_{0}\right) \quad(\forall i) \\
\Longrightarrow & 1-C\left(x_{i},\left(1+\varepsilon_{1}\right) \delta_{0}\right) \geq 1-C\left(x_{i},\left(1+\varepsilon_{2}\right) \delta_{0}\right)(\forall i) \\
\Longrightarrow & C^{*}\left(x_{i},\left(1+\varepsilon_{1}\right) \delta_{0}\right) \geq C^{*}\left(x_{i},\left(1+\varepsilon_{2}\right) \delta_{0}\right), \quad(\forall i)
\end{aligned}
$$

from whence, we obtain $\lambda_{\mathcal{X}}\left(\varepsilon_{1}\right) \geq \lambda_{\mathcal{X}}\left(\varepsilon_{2}\right)$, since avg is a monotonic operation.

\subsection{Suitability of Distance Functions based on $\lambda_{\rho}$}

Once again, from Figs. 4 (a) - (c), the rate of descent of $\lambda_{\rho}$ does indicate the rate of concentration. The faster it falls, the more is the concentration. For instance, from the above plots, it does appear that Fractional distances $\left(\mathcal{F}_{.04}\right)$ concentrate at a much slower rate than the other distance functions considered.

However, before comparing distance functions, perhaps an even more fundamental question that needs to be addressed is the following: Given a workload, what is a suitable distance function and how does $\lambda_{\rho}$ enable us to identify it? It is clear that, in the context of this work, a distance function $\rho$ is suitable if it does not concentrate much. In other words, a $\rho$ is suitable if all pairwise distances do not converge around a single value and provide good contrast. Since $\lambda_{\rho}$ measures this in terms of nearest neighbour (NN) distances, what is expected of a suitable distance function is that the NN distances of a large number of data points should be far less compared to that of the largest $\mathrm{NN}$ distance ${ }^{1}$. In the following, we attempt to place this intuitive idea in a more formal setting.

Definition 4.2. Let $\left(\Omega, \mathcal{X}, \rho, \mu_{\mathcal{X}}^{*}\right)$ be a given similarity workload and $\lambda_{\rho}$ be the corresponding dispersion function. We define $\varepsilon_{\rho}^{+}, \varepsilon_{\rho}^{-}$as follows:

$$
\begin{aligned}
& \varepsilon_{\rho}^{+}=\sup \left\{\varepsilon \in[-1, \infty) \mid \lambda_{\rho}(\varepsilon)=1\right\} \\
& \varepsilon_{\rho}^{-}=\inf \left\{\varepsilon \in[-1, \infty) \mid \lambda_{\rho}(\varepsilon)=0\right\}
\end{aligned}
$$

In other words, $\varepsilon_{\rho}^{+}, \varepsilon_{\rho}^{-}$are the values at which the dispersion function $\lambda_{\rho}$ begins and completes its descent. As we show below, both the point of decrease and the interval length play a role in classifying a distance function as suitable or not for a given workload.

Based on $\varepsilon_{\rho}^{+}, \varepsilon_{\rho}^{-}$the following observations can be made:

- Clearly, $\varepsilon_{\rho}^{+} \leq 0$. To see this, let $x_{0} \in \mathcal{X}$ be the data point that has the maximum $\mathrm{NN}$ distance, i.e., its nearest neighbour is at a distance $\delta_{0}$. Hence, for any $\varepsilon>0$, we have $C\left(x_{0},(1+\varepsilon) \delta_{0}\right)>1$, since $x_{0}$ contains (at least) its nearest neighbour and hence $\lambda_{\rho}(\varepsilon) \neq 1$.

- If $\rho$ is a suitable distance function, i.e., $\rho$ does not concentrate much for the given workload, then clearly $\delta_{0} \gg \delta_{i}$ for a large portion of the data set $\mathcal{X}$. This would imply that even for $\varepsilon \in[-1,0),(1+\varepsilon) \delta_{0}>\delta_{i}$ for a large portion of the data set and hence $\varepsilon_{\rho}^{+} \ll 0$.

- Let $\lambda_{\rho}$ be a slowly decreasing function and hence the interval $\left[\varepsilon^{+}, \varepsilon^{-}\right]$is large. This would mean that even for $\varepsilon \gg 0$ a large number of data points do not capture much of the rest of the data set in their $(1+\varepsilon)-\delta_{0}$ neighbourhood. Or equivalently, that their $(1+\varepsilon)$ $\delta_{0}$ count is much lesser than $N$ indicating that $\rho$ is still able to provide a good contrast for the considered workload and hence $\rho$ is a suitable distance function.

1. Note that, in the presence of outliers or in noisy data, $\delta_{0}$ can dominate other $\delta_{i}$ and make $\lambda_{\rho}$ less interpretable. However, in such scenarios, in some sense, one could contend that there is no concentration of distances. Further, taking $\delta_{0}$ to be any other internal operation of the $\delta_{i}$ 's, say for instance $\operatorname{avg}\left(\delta_{i}\right)$ did not seem to change the trend of the $\lambda_{\rho}$ curves, especially in the context of comparing distances based on $\lambda_{\rho}$. 


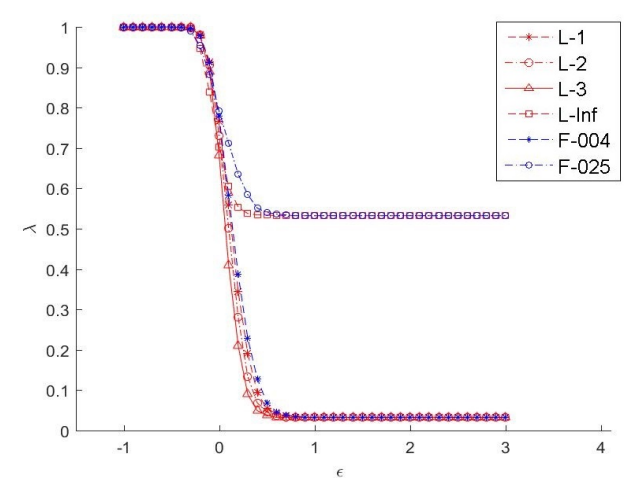

(a) Colon Cancer, $N=62, m=2000$

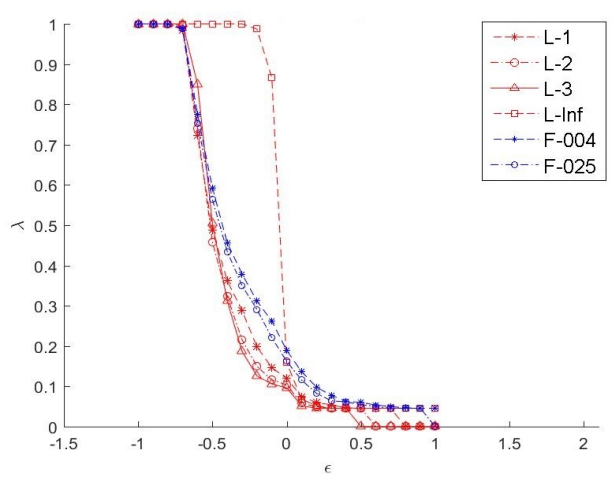

(c) Duke, $N=44, m=7129$

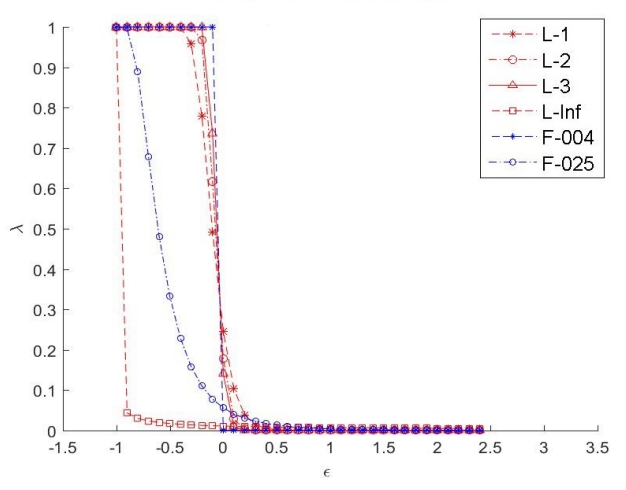

(b) Gisette, $N=6000, m=5000$

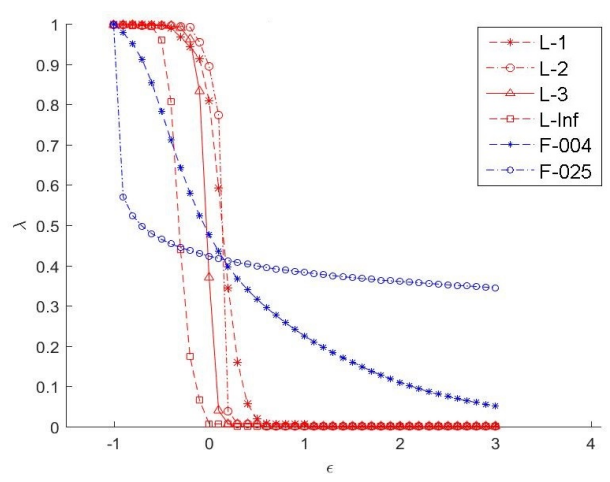

(d) Dexter, $N=300, m=20000$

Fig. 5. Plot of $\lambda_{\rho_{i}}$ for some UCI data sets listed in Table 3

- Let us consider a workload in high dimensions with a distance function that is known to concentrate. Then $\delta_{0} \approx \delta_{i}$ for almost every data point $x_{i}$ and hence

$$
\begin{aligned}
C\left(x_{i},(1+\varepsilon) \delta_{0}\right) & =\frac{1}{N} \approx C\left(x_{i},(1+\varepsilon) \delta_{i}\right), \text { and } \\
C^{*}\left(x_{i},(1+\varepsilon) \delta_{0}\right) & \approx C^{*}\left(x_{i},(1+\varepsilon) \delta_{i}\right)=\frac{N-1}{N} \approx 1 .
\end{aligned}
$$

Thus, $\lambda_{\rho}$ is almost a constant at 1 for $\varepsilon \in[-1,0)$, i.e., $\varepsilon_{\rho}^{+} \approx 0$. Further, even for small values of $\varepsilon>0$, $(1+\varepsilon) \delta_{0}>\delta_{i}$ for a large number of data points $x_{i}$ and hence the rate of decrease of $\lambda_{\rho}$ is very steep.

Thus, given a similarity workload, a suitable distance function $\rho$ is such that either $\varepsilon_{\rho}^{+} \ll 0$ or the interval $\left[\varepsilon_{\rho}^{+}, \varepsilon_{\rho}^{-}\right]$ is large, while if both $\varepsilon_{\rho}^{+} \approx 0$ and the interval $\left[\varepsilon_{\rho}^{+}, \varepsilon_{\rho}^{-}\right]$is small, it shows that the $\rho$ concentrates for $\mathcal{W}$ and is not so suitable.

Note that both these phenomena are noticeable from Fig. 4, where at low dimensions $\lambda_{\rho}$ does begin to decrease when $\varepsilon \in[-1,0)$ but at a slower rate (see Figs. 4(a) \& (b)), while at higher dimensions $\lambda_{\rho}$ is almost a constant at 1 and dips steeply (see Figs. 4(c) ). Further, from Table 4, the following can be observed:

(i) At low dimensions $(m=10)$, the $\varepsilon_{\rho}^{+}$values are far lesser than zero, in fact, $\varepsilon_{\rho}^{+}$values are closer to -1 .
Also the intervals $\left[\varepsilon_{\rho}^{+}, \varepsilon_{\rho}^{-}\right]$are large, indicating that all the considered distance functions seem suitable.

(ii) At medium dimensions $(m=100)$, we see a gradual shift in the $\varepsilon_{\rho}^{+}$values, which are now closer to zero than -1 . We also see a shrinking in the lengths of the corresponding $\left[\varepsilon_{\rho}^{+}, \varepsilon_{\rho}^{-}\right]$intervals.

(iii) At high dimensions $(m=1000)$, the behaviour of $\lambda_{\rho}$ is way different. For all the distance functions, both the $\varepsilon_{\rho}^{+} \approx 0$ and the lengths of the corresponding $\left[\varepsilon_{\rho}^{+}, \varepsilon_{\rho}^{-}\right]$intervals are small. Clearly, this indicates that all the 6 distance functions seem not so suitable for the considered workloads.

Based on Table 4 above, our analysis shows that Fractional distance functions $\left(\mathcal{F}_{.04}\right)$ concentrate at a much slower rate than other Minkowski distance functions. This observation is in tune with what many studies have reported earlier, see, for instance, [3]: that smaller values of $p$ in the Minkowski distance functions seem to concentrate less. In fact, as we show in Section 5.1, the values in Table 6 for some real workloads also seem to confirm their claim.

\subsection{Relation between $\alpha_{\rho}$ and $\lambda_{\rho}$}

From the above discussion, it does appear that $\alpha_{\rho}$ and $\lambda_{\rho}$ exhibit some similarities. In fact, comparing $\lambda_{\rho}$ and $\alpha_{\rho}$ the following commonalities can be observed: 
TABLE 4

Suitability of Distance functions on the basis of $\lambda_{\rho}$ - Synthetic Workloads

\begin{tabular}{|c|c|c|c|c|c|c|c|c|c|}
\hline Dataset & $m$ & $N$ & Indices & $\mathcal{F}_{0.04}$ & $\mathcal{F}_{0.25}$ & $\mathcal{L}_{1}$ & $\mathcal{L}_{2}$ & $\mathcal{L}_{3}$ & $\mathcal{L}_{\infty}$ \\
\hline \multirow{3}{*}{ Gaussian } & \multirow{3}{*}{10} & \multirow{3}{*}{1000} & $\varepsilon_{\rho}^{+}$ & -0.7 & -0.8 & -0.7 & -0.8 & -0.8 & -0.7 \\
\hline & & & $\varepsilon_{\rho}^{-}$ & 2.9 & 1.9 & 1.2 & 1. & 1. & 1.3 \\
\hline & & & {$\left[\varepsilon_{\rho}^{+}, \varepsilon_{\rho}^{-}\right]$} & 3.6 & 2.7 & 1.9 & 1.8 & 1.8 & 2.0 \\
\hline \multirow{3}{*}{ Gaussian } & \multirow{3}{*}{100} & \multirow{3}{*}{10000} & $\varepsilon_{\rho}^{+}$ & -0.4 & -0.4 & -0.4 & -0.3 & -0.3 & -0.4 \\
\hline & & & $\varepsilon_{\rho}^{-}$ & 0.5 & 0.4 & 0.3 & 0.2 & 0.2 & 0.7 \\
\hline & & & {$\left[\varepsilon_{\rho}^{+}, \varepsilon_{\rho}^{-}\right]$} & 0.9 & 0.8 & 0.7 & 0.5 & 0.5 & 1.1 \\
\hline \multirow{3}{*}{ Uniform } & \multirow{3}{*}{1000} & \multirow{3}{*}{10000} & $\varepsilon_{\rho}^{+}$ & -0.1 & -0.1 & -0.1 & -0.1 & -0.1 & -0.1 \\
\hline & & & $\varepsilon_{\rho}^{-}$ & 0.1 & 0.1 & 0.1 & 0.0 & 0.0 & 0.0 \\
\hline & & & {$\left[\varepsilon_{\rho}^{+}, \varepsilon_{\rho}^{-}\right]$} & 0.2 & 0.2 & 0.2 & 0.1 & 0.1 & 0.1 \\
\hline
\end{tabular}

(i) Both $\alpha_{\rho}$ and $\lambda_{\rho}$ not only illustrate but also can measure the rate of concentration of distance functions.

(ii) Unlike $\xi_{\rho}$ and $\gamma_{\rho}$, which indicate the presence of concentration as a function of or dependent on increase in dimensions, both $\alpha_{\rho}$ and $\lambda_{\rho}$ make use of the internal, and hence fixed, characteristics of the workload to do the same.

(iii) Both $\alpha_{\rho}$ and $\lambda_{\rho}$ are non-increasing functions and hence can offer insightful comparisons on the rate of concentration of different distance functions for an arbitrary but fixed workload under consideration.

However, $\alpha_{\rho}$ and $\lambda_{\rho}$ are not without their share of differences as enumerated below:

(i) $\alpha_{\rho}$ is a purely theoretical index while $\lambda_{\rho}$ is an empirical index.

(ii) Unless the underlying distribution is known, calculation of $\alpha_{\rho}$ is not possible, whereas $\lambda_{\rho}$ can still be determined. Hence $\lambda_{\rho}$ can be applied on real data sets to glean some useful information on the distances that could be considered when applying data analysis algorithms on them. This was already seen in Section 4.2. Also see Section 5.

(iii) In fact, the suitability of $\alpha_{\rho}$ to be employed as an empirical measure in practice is largely questionable. For instance, calculating $\alpha_{\rho}$ even for smaller data sets is extremely cumbersome. Recall that to find subsets with measure greater than $\frac{1}{2}$ requires $\sum_{k=\frac{N}{2}}^{N} C_{k} \approx 2^{N-1}$ computations. However, to evaluate $\lambda_{\rho}$ one only needs to work with $N$ singleton subsets. In fact, the computational complexity of determining $\alpha_{\rho}$ is of $\mathcal{O}\left(N 2^{N-1}\right)$, while that of $\lambda_{\rho}$ is only of $\mathcal{O}\left(N^{2} \log N\right)$. See the Appendix for more details. Thus determination of $\lambda_{\rho}$ is computationally far less expensive than $\alpha_{\rho}$.

(iv) However, an advantage that $\alpha_{\rho}$ enjoys that is not available to $\lambda_{\rho}$ is the following: $\alpha_{\rho}$ - being a theoretical index - is amenable for theoretical analysis, for instance, to obtain lower and upper bounds for a given workload when the underlying measurable metric space $(\Omega, \rho, \mu)$ is well defined. See [24] for some interesting existing results.

While the above discussion was based largely on empirical observations, the question that naturally arises is the following: Is there a relation between $\lambda_{\rho}$ and $\alpha_{\rho}$ ? The following result shows that, given a similarity workload where the distance $\rho$ is known to concentrate, $\lambda$ remains an upper bound of $\alpha$.

Recall, from Section 4.2, that if a $\rho$ concentrates, then both $\varepsilon_{\rho}^{+} \approx 0$ and the interval $\left[\varepsilon^{+}, \varepsilon^{-}\right]$is narrow. Further, since, $\delta_{0} \approx \delta_{i}$ for most of the data points $x_{i}$, from (9)

$$
\max _{x_{i} \in \mathcal{X}} C^{*}\left(x_{i},(1+\varepsilon) \delta_{0}\right) \approx \underset{x_{i} \in \mathcal{X}}{\operatorname{avg}} C^{*}\left(x_{i},(1+\varepsilon) \delta_{0}\right) .
$$

Theorem 4.3. Let $\left(\Omega, \mathcal{X}, \rho, \mu_{\mathcal{X}}^{*}\right)$ be a given similarity workload, where $\mathcal{X} \subset \Omega$ is finite and $\mu_{\mathcal{X}}^{*}$ is the normalised counting measure and the distance function $\rho$ is known to concentrate for this workload. Let $\varepsilon \in[-1, \infty)$ and $\delta_{0}$ be as defined in $(5)$. Let us denote by $r=(1+\varepsilon) \delta_{0}$. Then,

(i) $\alpha_{\rho}(r) \leq \lambda_{\rho}\left(\frac{r}{\delta_{0}}-1\right)$.

(ii) $\alpha_{\rho}(r)=0$, for any $r>r^{-}$, where $r^{-}=\left(1+\varepsilon_{\rho}^{-}\right) \delta_{0}$.

Proof: Firstly, note that $r$ is a function of $\varepsilon$ and hence as $\varepsilon$ varies from $[-1, \infty)$, we have that $r$ varies over $[0, \infty)=\overline{\mathbb{R}}_{+}$and hence $\alpha_{\rho}(r)$ is well-defined.

(i): Let $\varepsilon \in[-1, \infty)$ be arbitrary but fixed and $r$ be as defined above. Let $\mathcal{A}$ be the collection of all the subsets of $\mathcal{X}$ having measure greater than half, i.e.,

$$
\mathcal{A}=\left\{A \subset \mathcal{X}: \mu_{\mathcal{X}}^{*}(A) \geq \frac{1}{2}\right\}
$$

Let $A_{r}=\{x \in \mathcal{X}: \rho(x, a) \leq r$ for any $a \in A\}$, be the $r$-neighborhood of $A$ for $r \geq 0$. Since $(1+\varepsilon) \delta_{i} \leq(1+\varepsilon) \delta_{0}$ for every $\varepsilon \in[-1, \infty)$ and $i=1,2, \ldots, n$, for any arbitrary but fixed $A \in \mathcal{A}$ and for every $x_{i} \in A$, we have

$$
\begin{aligned}
& \mathrm{N}\left(x_{i},(1+\varepsilon) \delta_{i}\right) \subset \mathrm{N}\left(x_{i},(1+\varepsilon) \delta_{0}\right) \subset A_{r} \\
& \Longrightarrow \mu_{\mathcal{X}}^{*}\left(\mathrm{~N}\left(x_{i},(1+\varepsilon) \delta_{i}\right)\right) \leq \mu_{\mathcal{X}}^{*}\left(\mathrm{~N}\left(x_{i},(1+\varepsilon) \delta_{0}\right)\right) \leq \mu_{\mathcal{X}}^{*}\left(A_{r}\right) \\
& \Longrightarrow C\left(x_{i},(1+\varepsilon) \delta_{i}\right) \leq C\left(x_{i},(1+\varepsilon) \delta_{0}\right) \leq \mu_{\mathcal{X}}^{*}\left(A_{r}\right) \quad(\forall i) \\
& \Longrightarrow \mathcal{C}_{A}=\min _{x_{i} \in A} C\left(x_{i},(1+\varepsilon) \delta_{0}\right) \leq \mu_{\mathcal{X}}^{*}\left(A_{r}\right) .
\end{aligned}
$$

Now, since

$\inf _{A \in \mathcal{A}} \mathcal{C}_{A}=\inf _{A \in \mathcal{A}}\left\{\min _{x_{i} \in A} C\left(x_{i},(1+\varepsilon) \delta_{0}\right)\right\}=\min _{x_{i} \in \mathcal{X}} C\left(x_{i},(1+\varepsilon) \delta_{0}\right)$, 
and from (10), we have the following implications:

$$
\begin{aligned}
& \min _{x_{i} \in \mathcal{X}} C\left(x_{i},(1+\varepsilon) \delta_{0}\right) \approx \underset{x_{i} \in \mathcal{X}}{\operatorname{avg}} C\left(x_{i},(1+\varepsilon) \delta_{0}\right) \leq \inf _{A \in \mathcal{A}} \mu_{\mathcal{X}}^{*}\left(A_{r}\right) \\
& \Longrightarrow 1-\left(\min _{x_{i} \in \mathcal{X}} C\left(x_{i},(1+\varepsilon) \delta_{0}\right)\right) \geq 1-\inf _{A \in \mathcal{A}}\left\{\mu_{\mathcal{X}}^{*}\left(A_{r}\right)\right\} \\
& \Longrightarrow \max _{x_{i} \in \mathcal{X}}\left(1-C\left(x_{i},(1+\varepsilon) \delta_{0}\right)\right) \geq \sup _{A \in \mathcal{A}}\left\{\mu_{\mathcal{X}}^{*}\left(A_{r}^{c}\right)\right\} \\
& \Longrightarrow \max _{x_{i} \in \mathcal{X}}\left\{C^{*}\left(x_{i},(1+\varepsilon) \delta_{0}\right)\right\} \geq \sup _{A \in \mathcal{A}}\left\{\mu_{\mathcal{X}}^{*}\left(A_{r}^{c}\right)\right\} \\
& \Longrightarrow \max _{x_{i} \in \mathcal{X}}\left\{C^{*}\left(x_{i}, r\right)\right\} \geq \sup _{A \in \mathcal{A}}\left\{\mu_{\mathcal{X}}^{*}\left(A_{r}^{c}\right)\right\} \\
& \Longrightarrow \lambda_{\rho}(\varepsilon)=\lambda_{\rho}\left(\frac{r}{\delta_{0}}-1\right) \geq \alpha_{\rho}(r) \\
& (\text { ii) }: \text { Follows from part }(\mathrm{i}) .
\end{aligned}
$$

Remark 4.4.

(i) From Theorem 4.3, clearly $\lambda_{\rho}$ forms an upper bound for $\alpha_{\rho}$. Hence, for a given workload, if $\lambda_{\rho}$ itself falls very steeply to 0 , then $\alpha_{\rho}$ will fall faster, which indicates that the rate of concentration of the distance function under consideration will be very high.

(ii) It should also be noted that $\lambda_{\rho}$ is a tighter tail bound for $\alpha_{\rho}$ and is largely a loose bound for smaller values of $\varepsilon$, i.e., as $r$, equivalently $\varepsilon$ increases, $\lambda_{\rho}$ bounds $\alpha_{\rho}$ tighter. In fact, if $\rho$ is known to concentrate, then $\alpha_{\rho}$ falls steeply between $r^{+}=\left(1+\varepsilon_{\rho}^{+}\right) \delta_{0}$ and $r^{-}=(1+$ $\left.\varepsilon_{\rho}^{-}\right) \delta_{0}$.

(iii) Also note that, if $\lambda_{\rho}$ falls at a slower rate, i.e., the interval $\left[\varepsilon_{\rho}^{+}, \varepsilon_{\rho}^{-}\right]$is large, no conclusions can be made on $\alpha_{\rho}$ and hence on whether the distance function under consideration concentrates or not.

\section{Comparing Distance Functions using $\lambda_{\rho}$}

In Section 4.2, we discussed how to recognise the suitability of a distance function $\rho$ for a given workload based on its dispersion function $\lambda_{\rho}$. However, let us be given a workload and two distance functions $\rho_{1}, \rho_{2}$ whose dispersion functions, viz., $\lambda_{\rho_{1}}, \lambda_{\rho_{2}}$, show similar trends, say for instance, $\varepsilon_{\rho_{1}}^{+} \approx \varepsilon_{\rho_{2}}^{+}$and / or the lengths of the intervals $\left[\varepsilon_{\rho_{1}}^{+}, \varepsilon_{\rho_{1}}^{-}\right]$ and $\left[\varepsilon_{\rho_{2}}^{+}, \varepsilon_{\rho_{2}}^{-}\right]$could be the same. It is not yet clear how to compare them based on these parameters.

Further, one may not always be able to give an ordering (say, the usual point-wise ordering of functions) between $\lambda_{\rho_{1}}$ and $\lambda_{\rho_{2}}$, see for instance, Figs. 5 (a) and (d). Earlier, from Figs. 4 (a) - (c), based on the rate of descent of $\lambda_{\rho}$ we surmised that it does appear that Fractional distances $\left(\mathcal{F}_{\text {.04 }}\right)$ concentrate at a much slower rate than the other distance functions considered, which also coincided with many earlier studies that reported that smaller values of $p$ in the Minkowski distances seem to concentrate less. However, note that the above observation is purely based on visual illustrations. Also, we do not yet have a satisfactory result, á la if $0<p<q<\infty$ then $\lambda_{\mathcal{L}_{p}}<\lambda_{\mathcal{L}_{q}}$, either empirically or theoretically. In addition, the rate of decrease of $\lambda_{\rho}$ can be different over different intervals.

Nevertheless, our motivation is to propose an empirical index that would not only visually illustrate and measure the rate of concentration, but also allow us to compare between different distance functions on a given workload.
Towards this end, given a workload $\left(\Omega, \mathcal{X}, \rho, \mu_{\mathcal{X}}^{*}\right)$, we propose another empirical index $\tau_{\rho}$ that assigns a real value to every distance function $\rho$ based on $\lambda_{\rho}$, with the help of the parameters $\varepsilon_{\rho}^{+}, \varepsilon_{\rho}^{-}$proposed in Definition 4.2.

Definition 5.1. Given a similarity workload $\left(\Omega, \mathcal{X}, \rho, \mu_{\mathcal{X}}^{*}\right)$ and the corresponding dispersion function $\lambda_{\rho}$, let us define the index $\tau_{\rho}$ as follows:

$$
\tau_{\rho}=\int_{\varepsilon_{\rho}^{+}}^{\varepsilon_{\rho}^{-}} \lambda_{\rho}(\varepsilon) d \varepsilon .
$$

It is clear that $\tau_{\rho}$ calculates the area under $\lambda_{\rho}$ over the interval $\left[\varepsilon_{\rho}^{+}, \varepsilon_{\rho}^{-}\right]$. In the discrete case, with uniform step size for $\varepsilon, \tau_{\rho}$ can be calculated as $\tau_{\rho}=\sum_{\varepsilon=\varepsilon_{\rho}^{+}}^{\varepsilon_{\rho}^{-}} \lambda_{\rho}(\varepsilon)$.

Table 5 tabulates the $\tau_{\rho_{i}}$ values for the synthetic workloads and distance functions $\rho_{i}$ considered in Section 4.1. It is clear from the descending order of values of $\tau_{\rho_{i}}$ that the observation/claim made in earlier works that Fractional distances $\left(\mathcal{F}_{.04}\right)$ concentrate at a much slower rate still seems to hold true.

In Fig. 6, we give the calculated $\tau_{\rho}$ values (at the $i$-the iteration) and its variance (upto the $i$-the iteration) for $\rho=$ $\mathcal{L}_{1}, \mathcal{L}_{2}, \mathcal{L}_{\infty}$, based on repeated sampling (100 realisations) from the same high-dimensional distribution $\mathcal{X} \sim \mathcal{U}(0,1)^{m}$ with $m=100$ and $N=10,000$. The relatively small and almost constant values of the variance of $\tau_{\rho}$ shows that concentration of distances is rather a stable phenomenon in high dimensions and that $\tau_{\rho}$ does present one way of determining it consistently.

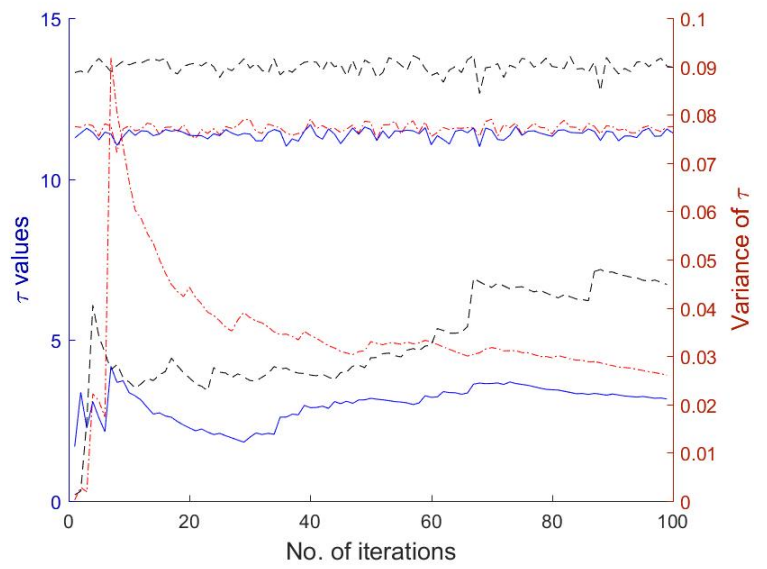

Fig. 6. $\tau_{\rho}$ values and its variance for the following distance functions, viz., $\mathcal{L}_{1}(-\cdot-), \mathcal{L}_{2}(-)$ and $\mathcal{L}_{\infty}(--)$.

While the index $\tau_{\rho}$ does induce an ordering on the considered distance functions, the question that arises now is this: Can it provide any more interesting or revealing information other than endorsing some general claims? What role does the ordering based on $\tau_{\rho}$ play in the algorithms applied on these real workloads? In the next section, we try to address these questions.

\subsection{Ordering based on Class Variable Accuracy}

Aggarwal et al. [3], based on their study of the concentration of Minkowski distances $\mathcal{L}_{p}$, strongly advocated the use 
TABLE 5

Comparing Distance functions on the basis of $\tau_{\rho}$ - Synthetic workloads

\begin{tabular}{|c|c|c|c|c|c|c|c|c|}
\hline Dataset & $m$ & $N$ & $\mathcal{F}_{0.04}$ & $\mathcal{F}_{0.25}$ & $\mathcal{L}_{1}$ & $\mathcal{L}_{2}$ & $\mathcal{L}_{3}$ & $\mathcal{L}_{\infty}$ \\
\hline Gaussian & 10 & 1000 & 18.04 & 14.92 & 11.02 & 11.20 & 11.00 & 10.76 \\
\hline Gaussian & 100 & 10000 & 7.12 & 6.42 & 5.54 & 4.38 & 4.22 & 7.10 \\
\hline Uniform & 1000 & 10000 & 2.54 & 2.89 & 2.02 & 1.99 & 1.97 & 2.00 \\
\hline
\end{tabular}

of these distances where the parameter $p \in(0,1)$ instead of the usual $p \in[1, \infty)$.

Towards empirical validation of their claim, they considered the following UCI data sets, given in Table 6, i.e., the sets of workloads $\left(\mathcal{X}_{i}, \rho_{j}, \mu_{\mathcal{X}}^{*}\right)$, where $\rho_{j}, j=1,2, \ldots, 7$ are the following Minkowski distance functions with $p=$ $0.1,0.5,1,2,4,10, \infty$. They determine the 'Class Variable Accuracy' $\beta_{i j}$ for each of the above workloads as follows: data.

Step 1: Remove the class label information from the

Step 2: Fix an $x_{k} \in \mathcal{X}_{i}$ and search for $\ell$ nearest neighbours based on $\rho_{j}$.

Step 3: Among these $\ell$ neighbours count those that belong to the same class as $x_{k}$, say $\beta_{i j}^{k}$.

Step 4: Repeat Step 3 for all $x_{k} \in \mathcal{X}_{i}$ and find $\beta_{i j}=$ $\sum_{k} \beta_{i j}^{k}$.

Step 5: Repeat Steps $2-4$ for different $\rho_{j}$.

Step 6: Repeat Steps $2-5$ for different data sets $\mathcal{X}_{i}$.

Based on the decreasing order of $\beta_{i j}$, they ranked the above 7 distance functions as follows:

$$
\mathcal{F}_{0.1} \succ \mathcal{F}_{0.5} \succ \mathcal{L}_{1} \succ \mathcal{L}_{2} \succ \mathcal{L}_{4} \succ \mathcal{L}_{10} \succ \mathcal{L}_{\infty} .
$$

In Table 6 we present the corresponding $\tau_{\rho}$ values for these work loads. Clearly from the $\tau_{\rho}$ values we observe that the ordering given in (12) is exactly the one obtained for descending values of $\tau_{\rho}$.

\subsection{Comparison of Secondary Distance Measures}

So far, we have considered only the Minkowski norms. Recently there have been attempts to employ Secondary Distance measures, so called since these are themselves derived from other primary distance measures, typically the Minkowski metrics. These have been shown to work well, especially, in mitigating some aspects of DC in many Machine Learning problems like clustering and classification, see for instance, [17], [13], [33], [34].

We consider the following five secondary distance measures in this work, viz., the Shared Nearest Neighbour (SNN) [17], Local Scaling (LS) [38], Mutual Proximity (MP) [30], Non-iterative Contextual Dissimilarity Measure (NICDM) [22] and Hubness-aware SNN (HSNN) [35].

\subsubsection{On Some Synthetic Data Sets}

In this section, we consider the 10 synthetic datasets employed in [35]. These are high-dimensional Gaussian mixture data with high class overlap. Each of these data sets is 100-dimensional with 10 -classes consisting of more than a thousand points each ${ }^{2}$. According to [35] these data sets exhibit substantial hubness and hence are very difficult for $k$-NN classification.

2. Available at http://ailab.ijs.si/nenad_tomasev/datasets/

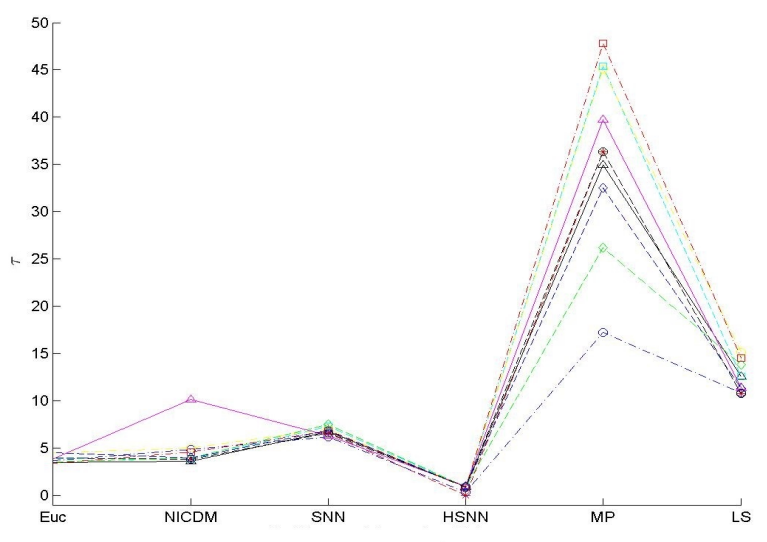

Fig. 7. Plot of $\tau_{\rho_{i}}$ for the 10 Synthetic Datasets considered in [35]

The $\tau_{\rho}$ values were calculated for each of the 10 data sets and all the above five secondary distance measures, with the primary distance being the Euclidean distance. The superimposed $\tau_{\rho}$ values for the above 6 distance measures is given in Fig. 7. Larger the $\tau_{\rho}$ value greater is the suitability of the distance function $\rho$. It is clear that our studies also validate the arguments of the authors in [35] that secondary distances demonstrate great potential in correcting hubness-related problems.

\subsubsection{On Real Data Sets}

In this section, we consider the 6 UCI data sets listed in Table 7, which were chosen not only for their high dimensionality but also since a few of them, due to their intrinsic hubness, have been employed in many works, see for instance [14], and hence provides for meaningful comparative analysis. Further, they also provide a good mix of datasets, where for Datasets $1 \& 2$ we have $N \gg m$, while for Datasets $3,5 \& 6, m \gg N$ and for Dataset 4 , $m \approx N$.

The $\tau_{\rho}$ values for the above secondary distances on these data sets are presented in Table 7 . The largest values are given in bold ${ }^{3}$.

In [14], the authors have made a comparative study of the suitability of three secondary distance functions, viz., MP, LS and SNN, on the Datasets $1,2,4$ \& 6 given in Table 7, w.r.t. their classification accuracy. It is worthy to note that the ranking of the distance functions based on $\tau_{\rho}$ and classification accuracy remain identical, except in the case of Dataset 1 where the ranks of MP and LS are

3. It is interesting to note that it does appear that distances that can handle one aspect of the Dimensionality Curse, say the hubness phenomenon or the CoD, may not necessarily be good at handling other aspects. 
TABLE 6

Comparing Minkowski Distance functions on the basis of $\tau_{\rho}$ - Some UCI Data sets considered in [3]

\begin{tabular}{|c|c|c|c|c|c|c|c|c|c|}
\hline Dataset & $m$ & $N$ & $\mathcal{F}_{0.10}$ & $\mathcal{F}_{0.50}$ & $\mathcal{L}_{1}$ & $\mathcal{L}_{2}$ & $\mathcal{L}_{4}$ & $\mathcal{L}_{10}$ & $\mathcal{L}_{\infty}$ \\
\hline Musk & 167 & 476 & 85.79 & 41.18 & 34.38 & 25.59 & 19.03 & 15.13 & 15.85 \\
\hline Breast Cancer WDBC & 30 & 569 & 11.62 & 10.58 & 6.54 & 6.54 & 6.37 & 6.23 & 6.20 \\
\hline Ionosphere & 34 & 351 & 18.79 & 11.46 & 8.46 & 8.09 & 9.28 & 11.34 & 13.84 \\
\hline Segment & 19 & 210 & 21.94 & 8.94 & 4.21 & 3.55 & 3.35 & 3.32 & 3.31 \\
\hline
\end{tabular}

TABLE 7

Comparing Secondary Distance functions on the basis of $\tau_{\rho}$ - Real workloads

\begin{tabular}{|c||c|c|c|c|c|c|}
\hline Dataset & $\mathcal{L}_{2}$ & NICDM & SNN & HSNN & MP & LS \\
\hline Splice & 6.69 & 2.42 & 9.67 & 0.48 & 52.73 & 15.46 \\
\hline Protein & 5.43 & 3.08 & 6.69 & 0.0009 & 10.4 & 20.85 \\
\hline Cancer & 5.77 & 20.51 & 34.75 & 18.05 & 39.26 & 25.17 \\
\hline Gisette & 1.8 & 2.77 & 10.12 & 1.23 & 84.117 & 7.87 \\
\hline Duke & 3.34 & 27.46 & 31.56 & 26.8 & 29.69 & 43.97 \\
\hline Dexter & 11.3 & 50.1 & 36.23 & 0.47 & 90.37 & 181.4 \\
\hline
\end{tabular}

interchanged ${ }^{4}$. We have used the same parameter values for all the secondary distance measures as employed in [14], i.e., $r=10$ in SNN, $q=10$ in LS and used $1-\cdot$ to obtain the distances from the similarities.

\section{Conclusion}

In this work, we began by discussing the concentration of distances phenomenon. Our examination of the different indices that either illustrate or measure this phenomenon revealed the need for an efficient empirical index that would not only illustrate but also measure the rate of concentration and enable comparison of distance functions with regards to their suitability in real workloads. With this as the motivation for this work, we have proposed a novel yardstick called the dispersion function $\lambda_{\rho}$ which is an empirical measure.

Based on the dispersion function, we have also introduced an index $\tau_{\rho}$ that allows us to compare distances with regards to their suitability in real workloads, thus helping us to achieve our twin objectives. Further exploration of both $\lambda_{\rho}$ and $\tau_{\rho}$ on some real workloads that have been used in existing studies seem to validate the usefulness of both the dispersion function $\lambda_{\rho}$ and the index $\tau_{\rho}$ in judging the suitability of a distance function for a given workload.

So far the theory of concentration of norms has been well studied and explored but always in a non-positive way. From Sections 2.3.1 and 2.3.2, we see that Euclidean norms and other Minkowski-type distances do not behave well in high dimension. In fact, we have that all the Minkowskitype distances concentrate, but only at differing rates. The current work differs from existing studies in the following important ways:

(i) To the best of the authors' knowledge, this is the first empirical index to measure the rate of concentration of a distance function, while other empirical indices only illustrate the effect and that too only as a function of increasing dimensions.

4. Note, however, that the classification accuracies of these two distances on Dataset 1 are almost the same at $77.2 \& 77.9$, respectively. (ii) This work not only tries to determine whether a distance function concentrates for a given workload, but also proposes an index to compare distances and suggest their suitability.

This work can, and should be, seen as yet another exploratory but a positive study on this phenomenon. Clearly, a more theoretical analysis of the dispersion function is in order, which we intend to take up in the near future.

\section{Appendix}

Complexity Considerations: $\alpha_{\rho}$ vs. $\lambda_{\rho}$

In this section we study the time complexity of calculating $\alpha_{\rho}$ vs. $\lambda_{\rho}$ for a given data set $\mathcal{X}$ and a distance function $\rho$.

Let $\mathcal{K}=\left\{A \subseteq \mathcal{X} \mid \sharp A \geq \frac{N}{2}\right\}$. Let us fix an $\epsilon>0$. For an $x \in \mathcal{X}$, let $\sharp\left(\left[\mathrm{N}_{\epsilon}(x)\right]^{C}\right)$ denote the number of elements that are at a distance greater than $\epsilon$ from $x$ w.r.t. $\rho$ and, further, for an $A \in \mathcal{K}$, let $A_{\epsilon}^{C}$ denote the complement of the $\epsilon$-dilation of $A$.

To calculate $\alpha_{\rho}(\epsilon)=\sup _{A \in \mathcal{K}}\left\{\sharp\left(A_{\epsilon}^{C}\right)\right\}$ we proceed as follows:

There are $\sum_{k=\frac{N}{2}}^{N}{ }^{N} C_{k}=2^{N-1}$ elements in $\mathcal{K}$ and hence finding $\mathcal{K}$ is of the order $2^{N-1}$.

Step 2: We calculate $\sharp\left(\left[\mathrm{N}_{\epsilon}(x)\right]^{C}\right)$ as follows:

- Sort the pairwise distance matrix, which is of $N^{2} \log N$ complexity.

- For the fixed $\epsilon>0$, for each $x \in \mathcal{X}$ - whose sorted pairwise distances to other data points is a row in the distance matrix - the complexity of determining $\sharp\left(\left[\mathrm{N}_{\epsilon}(x)\right]^{C}\right)$ is $N \log N$.

Step 3: Let us fix an $A \in \mathcal{K}$. Now the cardinality of $\epsilon$-dilation of $A$ is given by $\sharp\left(A_{\epsilon}^{C}\right)=\sum_{x \in A} \sharp\left(\left[\mathrm{N}_{\epsilon}(x)\right]^{C}\right)$. Hence the complexity of finding $\sharp\left(A_{\epsilon}^{C}\right)$ for each $A \in \mathcal{K}$ is $\sum_{k=\frac{N}{2}}^{N}{ }^{N} C_{k} \cdot k \approx N \cdot 2^{N-1}$

Step 4: Finally, the complexity of finding the $\sup _{A \in \mathcal{K}} \sharp\left(A_{\epsilon}^{C}\right)$ is of the order $2^{N-1}$.

Thus, on the whole, the complexity of calculating $\alpha_{\rho}(\epsilon)$, for a given $\epsilon>0$ is the sum of the above, viz., $2^{N-1}+$ $N^{2} \log N+N \log N+N 2^{N-1}+2^{N-1}$ and hence is of the order $\mathcal{O}\left(n \cdot 2^{N-1}\right)$.

From Definition 3.2, we have $\lambda_{\rho}(\varepsilon)=\underset{x_{i} \in \mathcal{X}}{\operatorname{avg}}\left\{C^{*}\left(x_{i},(1+\right.\right.$ $\left.\left.\varepsilon) \delta_{0}\right)\right\}=\underset{x_{i} \in \mathcal{X}}{\operatorname{avg}}\left\{\mathrm{N}_{(1+\varepsilon) \delta_{0}}(x)\right\}$. Its time complexity can be calculated as follows: 
Step 1: To find $\delta_{0}$, we once again sort the pairwise distance matrix and find the maximum of the column 2 which is of order $N^{2} \log N+N$.

Step 2: Finding $C^{*}\left(x,(1+\varepsilon) \delta_{0}\right)$ has the same complexity as that of finding $\mathrm{N}_{(1+\varepsilon) \delta_{0}}(x)$, which is $N \log N$.

Step 3: Now averaging over all $x \in \mathcal{X}$ is of linear order.

Thus the total time complexity of calculating $\lambda_{\rho}(\epsilon)$ is of the order $\mathcal{O}\left(N^{2} \cdot \log N\right)$. Note that the costliest step in this algorithm is that of calculating $\delta_{0}$. However, if one were to use an approximate nearest neighbour search method, then the complexity can be brought down considerably. For instance, if one employs the LSH, keeping the width parameter ' $k$ ' and the time taken for evaluation ' $t$ ' are kept fixed, its complexity is $O\left(N^{1+p}\right)$, with $p \ll 1$. Thus the overall complexity of calculating lambda is $O\left(N^{1+p}\right)$, which is near linear complexity.

\section{References}

[1] C. C. Aggarwal, "Re-designing distance functions and distancebased applications for high dimensional data," SIGMOD Record, vol. 30, no. 1, pp. 13-18, 2001

[2] —, "On the analytical properties of high-dimensional randomization," IEEE Trans. Knowl. Data Eng., vol. 25, no. 7, pp. 1628-1642, 2013.

[3] C. C. Aggarwal, A. Hinneburg, and D. A. Keim, "On the surprising behavior of distance metrics in high dimensional spaces," in Proc. ICDT 2001, London, UK, 2001, pp. 420-434.

[4] J. Aucouturier and F. Pachet, "A scale-free distribution of false positives for a large class of audio similarity measures," Pattern Recognition, vol. 41, no. 1, pp. 272-284, 2007.

[5] R. Bellmann, Adaptive Control Processes: A Guided Tour. Princeton Univ. Press, 1961.

[6] K. P. Bennett, U. M. Fayyad, and D. Geiger, "Density-based indexing for approximate nearest-neighbor queries," in Proc. 5th ACM SIGKDD, San Diego, USA,, 1999, pp. 233-243.

[7] S. Berchtold, B. Ertl, D. A. Keim, H. Kriegel, and T. Seidl, "Fast nearest neighbor search in high-dimensional space," in Proc. 14th ICDE, Orlando, Florida, USA,

[8] S. Berchtold, D. A. Keim, H. Kriegel, and T. Seidl, "Indexing the solution space: A new technique for nearest neighbor search in high-dimensional space," IEEE Trans. Knowl. Data Eng., vol. 12, no. 1 , pp. $45-57,2000$.

[9] K. S. Beyer, J. Goldstein, R. Ramakrishnan, and U. Shaft, "When is "nearest neighbor" meaningful?" in Proc. ICDT '99, Jerusalem, Israel,, 1999, pp. 217-235.

[10] E. Chávez, G. Navarro, R. A. Baeza-Yates, and J. L. Marroquín, "Searching in metric spaces," ACM Comput. Surv., vol. 33, no. 3, pp. 273-321, 2001.

[11] F. H. Croom, Principles of Topology. Thomson Learning Asia, 2002.

[12] R. J. Durrant and A. Kabán, "When is 'nearest neighbour' meaningful: A converse theorem and implications," J. Complexity, vol. 25, no. 4, pp. 385-397, 2009.

[13] A. Flexer and D. Schnitzer, "Can shared nearest neighbors reduce hubness in high-dimensional spaces?" in 13th IEEE ICDM Workshops, TX, USA, December 7-10, 2013, 2013, pp. 460-467.

[14] — , "Choosing $l^{P}$ norms in high-dimensional spaces based on hub analysis," Neurocomputing, vol. 169, pp. 281-287, 2015.

[15] D. François, V. Wertz, and M. Verleysen, "The concentration of fractional distances," IEEE Trans. Knowl. Data Eng., vol. 19, no. 7, pp. 873-886, 2007.

[16] S. Har-Peled, P. Indyk, and R. Motwani, "Approximate nearest neighbor: Towards removing the curse of dimensionality," Theory of Computing, vol. 8, no. 1, pp. 321-350, 2012.

[17] M. E. Houle, H. Kriegel, P. Kröger, E. Schubert, and A. Zimek, "Can shared-neighbor distances defeat the curse of dimensionality?" in Proc. SSDBM 2010, Heidelberg, Germany,, ser. Lecture Notes in Computer Science, M. Gertz and B. Ludäscher, Eds., vol. 6187. Springer, 2010, pp. 482-500.
[18] C.-M. Hsu and M.-S. Chen, "On the design and applicability of distance functions in high-dimensional data space," IEEE Trans. Knowl. Data Eng., vol. 21, no. 4, pp. 523-536, 2009.

[19] G. F. Hughes, "On the mean accuracy of statistical pattern recognizers," IEEE Transactions on Information Theory, vol. 14, no. 1 , pp. $55-63,1968$.

[20] P. Indyk and R. Motwani, "Approximate nearest neighbors: Towards removing the curse of dimensionality," in Proc. of the $30^{\text {th }}$ Annual ACM Symposium on the Theory of Computing, fTexas, USA, May 23-26, 1998, J. S. Vitter, Ed. ACM, 1998, pp. 604-613.

[21] B. Jayaram and F. Klawonn, "Can unbounded distance measures mitigate the curse of dimensionality?" Int. Jl. of Data Mining, Modelling and Mgmt., vol. 4, no. 4, pp. 361-383, 2012.

[22] H. Jegou, H. Harzallah, and C. Schmid, "A contextual dissimilarity measure for accurate and efficient image search," in IEEE (CVPR 2007), Minnesota, USA. IEEE Computer Society, 2007.

[23] F. Korn, B. Pagel, and C. Faloutsos, "On the 'dimensionality curse' and the 'self-similarity blessing'," IEEE Trans. Knowl. Data Eng., vol. 13, no. 1, pp. 96-111, 2001.

[24] M. Ledoux, The Concentration of Measure Phenomenon, ser. Mathematical Surveys and Monographs. American Mathematical Society, 2001, vol. 89.

[25] V. Pestov, "On the geometry of similarity search: Dimensionality curse and concentration of measure," Inf. Process. Lett., vol. 73 no. $1-2$, pp. $47-51,2000$.

[26] _ - "Intrinsic dimension of a dataset: what properties does one expect?" in IJCNN, 2007, pp. 2959-2964.

[27] — " "Is the k-NN classifier in high dimensions affected by the curse of dimensionality?" Computers \& Mathematics with Applications, vol. 65, no. 10, pp. 1427-1437, 2013.

[28] M. Radovanovic, A. Nanopoulos, and M. Ivanovic, "Nearest neighbors in high-dimensional data: the emergence and influence of hubs," in Proc. 26th ICML 2009, Montreal, Canada,, 2009, pp. $865-872$.

[29] _ - "Hubs in space: Popular nearest neighbors in highdimensional data," Journal of Machine Learning Research, vol. 11, pp. 2487-2531, 2010.

[30] D. Schnitzer, A. Flexer, M. Schedl, and G. Widmer, "Local and global scaling reduce hubs in space," Journal of Machine Learning Research, vol. 13, pp. 2871-2902, 2012.

[31] D. W. Scott, Multivariate Density Estimation. New Jersey: John Wiley and Sons, 2015.

[32] U. Shaft and R. Ramakrishnan, "When is nearest neighbors indexable?" in Database Theory - ICDT 2005, 10th International Conference, Edinburgh, UK, January 5-7, 2005, Proceedings, T. Eiter and L. Libkin, Eds., 2005, pp. 158-172.

[33] N. Tomasev, "Taming the empirical hubness risk in many dimensions," in Proc. SIAM ICDM 2015, Vancouver, Canada, S. Venkatasubramanian and J. Ye, Eds. SIAM, 2015, pp. 891899.

[34] N. Tomasev and K. Buza, "Hubness-aware knn classification of high-dimensional data in presence of label noise," Neurocomputing, vol. 160, pp. 157-172, 2015.

[35] N. Tomasev and D. Mladenic, "Hubness-aware shared neighbor distances for high-dimensional $\$ \$ \mathrm{k} \$ \$$-nearest neighbor classification," Knowl. Inf. Syst., vol. 39, no. 1, pp. 89-122, 2014.

[36] N. Tomasev, M. Radovanovic, D. Mladenic, and M. Ivanovic, "The role of hubness in clustering high-dimensional data," IEEE Trans. Knowl. Data Eng., vol. 26, no. 3, pp. 739-751, 2014.

[37] G. S. Vitali D. Milman, Asymptotic Theory of Finite Dimensional Normed Spaces, ser. Lecture Notes in Mathematics, B. E. A. Dold, Ed. Springer, 1986, vol. 1200.

[38] L. Zelnik-Manor and P. Perona, "Self-tuning spectral clustering," in NIPS 2004, Vancouver, Canada, 2004, pp. 1601-1608.

[39] Y. Zhai, Y. Ong, and I. W. Tsang, "The emerging "Big Dimensionality"," IEEE Comp. Int. Mag., vol. 9, no. 3, pp. 14$26,2014$. 


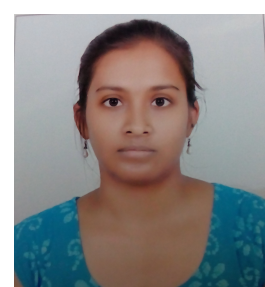

Sushma Kumari received her B.Sc. in Mathematics from Delhi University, India in 2013 and her masters from the Department of Mathematics, Indian Institute of Technology Hyderabad, India. Currently she is pursuing her doctoral studies at the Department of Mathematics, Kyoto University, Japan. Ms. Kumari's research interests include data analysis and randomized matrices.

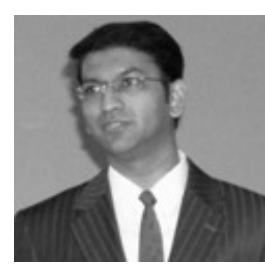

Balasubramaniam Jayaram (S'02-A'03M'04) received the M.Sc. and Ph.D. degrees in Mathematics from Sri Sathya Sai Institute of Higher Learning, India, in 1999 and 2004, respectively. He has been a visiting researcher at universities in Germany, Austria, Slovakia and Czech Republic. He is currently an Associate Professor at the Department of Mathematics, Indian Institute of Technology Hyderabad, India.

Dr. Jayaram's current research interests include fuzzy aggregation operations, approximate reasoning and issues in high dimensional data analysis. He has co-authored a research monograph on Fuzzy Implications and is the author or co-author of more than 60 published papers.

Dr. Jayaram is a member of EUSFLAT, IEEE and IEEE Computational Intelligence societies, and is an Experienced Research Fellow of the Alexander von Humboldt foundation.

Dr. Jayaram is an Associate Editor for the IEEE Transactions on Fuzzy Systems (2014-) and Fuzzy Sets and Systems (2016 - ). 